\title{
Time-Dependent Response Versus Scan Angle for MODIS Reflective Solar Bands
}

\author{
Junqiang Sun, Xiaoxiong Xiong, Amit Angal, Hongda Chen, Aisheng Wu, and Xu Geng
}

\begin{abstract}
The Moderate Resolution Imaging Spectroradiometer (MODIS) instruments currently operate onboard the National Aeronautics and Space Administration (NASA's) Terra and Aqua spacecraft, launched on December 18, 1999 and May 4, 2002, respectively. MODIS has 36 spectral bands, among which 20 are reflective solar bands (RSBs) covering a spectral range from 0.412 to $2.13 \mu \mathrm{m}$. The RSBs are calibrated on orbit using a solar diffuser (SD) and an SD stability monitor and with additional measurements from lunar observations via a space view (SV) port. Selected pseudo-invariant desert sites are also used to track the RSB on-orbit gain change, particularly for short-wavelength bands. MODIS views the Earth surface, SV, and the onboard calibrators using a two-sided scan mirror. The response versus scan angle (RVS) of the scan mirror was characterized prior to launch, and its changes are tracked using observations made at different angles of incidence from onboard SD, lunar, and Earth view (EV) measurements. These observations show that the optical properties of the scan mirror have experienced large wavelength-dependent degradation in both the visible and near infrared spectral regions. Algorithms have been developed to track the on-orbit RVS change using the calibrators and the selected desert sites. These algorithms have been applied to both Terra and Aqua MODIS Level 1B (L1B) to improve the EV data accuracy since L1B Collection 4, refined in Collection 5, and further improved in the latest Collection 6 (C6). In C6, two approaches have been used to derive the time-dependent RVS for MODIS RSB. The first approach relies on data collected from sensor onboard calibrators and mirror side ratios from EV observations. The second approach uses onboard calibrators and EV response trending from selected desert sites. This approach is mainly used for the bands with much larger changes in their time-dependent RVS, such as the Terra MODIS bands 1-4, 8, and 9 and the Aqua MODIS bands 8 and 9. In this paper, the algorithms of these approaches are described, their performance is demonstrated, and their impact on L1B products is discussed. In general, the shorter wavelength bands have experienced a larger on-orbit RVS change, which, in general, are mirror side and detector dependent. The on-orbit RVS change due to the degradation of band 8 can be as large as $35 \%$ for Terra MODIS and $20 \%$ for Aqua MODIS. Vital to maintaining the accuracy of the MODIS L1B products is an accurate characterization of the on-orbit RVS change. The derived time-independent RVS, implemented in C6, makes an important improvement to the quality of the MODIS L1B products.
\end{abstract}

Manuscript received March 14, 2013; revised May 22, 2013; accepted June 22, 2013.

J. Sun, H. Chen, A. Wu, and X. Geng are with the Sigma Space Corporation, Lanham, MD 20706 USA (e-mail: junqiang.sun@ sigmaspace.com).

$X$. Xiong is with the Sciences and Exploration Directorate, NASA/Goddard Space Flight Center, Greenbelt, MD 20771 USA.

A. Angal is with the Science Systems and Applications, Inc., Lanham, MD 20706 USA.

Color versions of one or more of the figures in this paper are available online at http://ieeexplore.ieee.org.

Digital Object Identifier 10.1109/TGRS.2013.2271448
Index Terms-Aqua, desert, Moderate Resolution Imaging Spectroradiometer (MODIS), moon, reflective solar bands (RSBs), response versus scan angle (RVS), scan mirror, solar diffuser (SD), Terra.

\section{INTRODUCTION}

$\mathbf{T}$ WO Moderate Resolution Imaging Spectroradiometer (MODIS) instruments are currently on orbit, one aboard the Terra spacecraft launched on December 18, 1999 and the other on the Aqua spacecraft launched on May 4, 2002 [1][4]. MODIS has 36 spectral bands ranging in wavelength from 0.412 to $14.4 \mu \mathrm{m}$. Continuous global observations are made at three nadir spatial resolutions: $0.25 \mathrm{~km}$ for bands 1 and 2, $0.5 \mathrm{~km}$ for bands 3-7, and $1 \mathrm{~km}$ for the bands 8-36 [5], [6]. The detectors of the 36 bands are distributed on four focal plane assemblies (FPAs): visible (VIS), near infrared (NIR), short-wave infrared/midwave infrared, and long-wave infrared. MODIS has four onboard calibrators in addition to a space view (SV) port. The calibrators are a solar diffuser (SD) with an SD stability monitor (SDSM) [7]-[9], a blackbody (BB) [10], [11], and a spectroradiometric calibration assembly (SRCA) [12], [13]. MODIS views the Earth surface, SV, and the onboard calibrators via a rotating two-side scan mirror.

The MODIS scan mirror is shown in Fig. 1. It is constructed from a polished nickel-plated beryllium base coated with highpurity silver, which is then overcoated with the Denton proprietary silicon monoxide and silicon dioxide mixture [14]. Using both sides of the scan mirror, MODIS collects data over five sectors, the SD, BB, SRCA, SV, and Earth view (EV), sequentially with a scan period of $1.477 \mathrm{~s}$. The light from each of these sectors is incident on the scan mirror at a different angle of incidence (AOI). The AOI varies from $10.5^{\circ}$ to $65.5^{\circ}$ for $\mathrm{EV}$ with the SD located at $50.2^{\circ}$, the $\mathrm{BB}$ at $26.2^{\circ}$, the SRCA (nadir) at $38.2^{\circ}$, and the $\mathrm{SV}$ at $11.2^{\circ}$ as shown in Fig. 2. The gain of the instrument at any AOI for a given band, detector, and mirror side (MS) depends on the reflectance of the MS at the AOI, the optical elements in its path, the electronic configuration, and the detector. Since the last three items are independent of the AOI of the scan mirror, the variation of the instrument gain with the AOI strongly depends on the reflectance of the scan mirror. A response versus scan angle (RVS) function is used to describe the dependence of the MODIS response on the AOI for each band, detector, and MS.

Among the 36 bands, bands $1-19$ and 26 are the reflective solar bands (RSBs). Their wavelengths and specifications are listed in Table I. The RSB RVS of the scan mirror for both MODIS instruments was characterized prior to launch by measuring the response at different AOIs using a spherical 


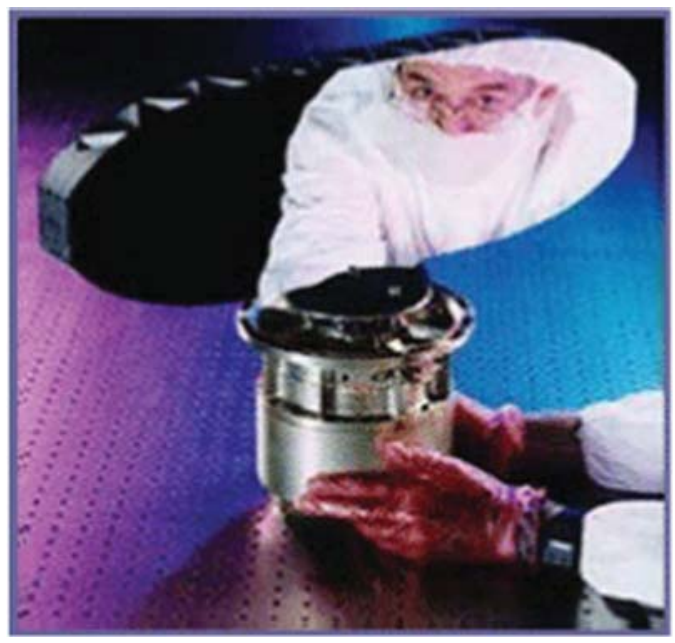

Fig. 1. MODIS scan mirror.

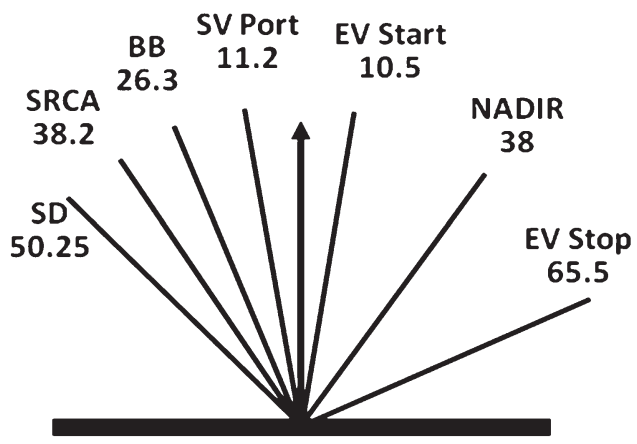

Fig. 2. AOIs for the MODIS scan mirror.

integrating source (SIS) [15]-[17]. The derived prelaunch RVS clearly indicates a dependence on both AOI and wavelength. The RSBs are calibrated on orbit by the SD with its degradation being tracked by the SDSM [7]-[9]. Both instruments are scheduled to view the Moon approximately monthly [18], [19]. The lunar calibration can be used to derive the RSB onorbit gain change at the AOI of the SV. MODIS has monthly SRCA measurements in radiometric mode, which provide some information for the RSB on-orbit gain change at the AOI of the nadir [12], [13]. The selected ocean sites and pseudoinvariant desert targets are also used to track the MS ratios and the response trending at various AOIs, respectively. The RVS on-orbit change can be derived using all the aforementioned measurements with approximation for the AOI dependence [20]-[24].

The time-dependent RSB RVS algorithms have been developed and applied in MODIS Level 1B (L1B) since Collection 4 (C4). The $\mathrm{C} 4$ algorithms were developed based solely on the measurements of the onboard calibrators, which also include lunar observations, and the time-dependent RVS was applied to short-wavelength bands exhibiting a relative large change in their responses. In MODIS L1B Collection 5 (C5), the algorithms were refined to include the EV MS response ratios in the derivation of the time-dependent RVS. In the latest MODIS L1B version, Collection 6 (C6), the on-orbit RVS is characterized using a combination of measurements from the onboard calibrators as used in $\mathrm{C} 5$ and $\mathrm{EV}$ response trending at other AOIs from pseudo-invariant desert targets [21]. For most MODIS RSBs with relatively small changes in their responses, the time-dependent RVS can still be derived using the onboard SD and lunar observations. In the case of short-wavelength RSB, the SD/SDSM and lunar observations alone may not provide an accurate estimation of the gain, and furthermore, the gain at other AOIs is required to determine the on-orbit RVS change. For these bands, the EV response trending over pseudo-invariant desert targets is included to derive the RVS. In this paper, the formulation of the MODIS C6 RSB RVS algorithms is presented, and the performance of the on-orbit RVS algorithms is discussed. Improvements in the L1B products with the time-dependent RVS derived using the on-orbit RVS algorithm are also demonstrated. The bands with shorter wavelength have exhibited larger RVS change on orbit. For certain wavelength bands with large RVS change, the RVS has also exhibited detector dependence, particularly for Terra MODIS. In spite of being identical instruments, Terra and Aqua MODIS have exhibited significantly different on-orbit behavior of the RVS change. In general, the Terra RSBs have experienced more change, primarily due to the SD door anomaly (July 2, 2003) whereby the SD door was set to a permanent open position. In addition, due to the smoking incident during the prelaunch characterization [8], the difference in the responsivity of the two MSs of Terra MODIS RSB has exhibited significant variation on orbit.

\section{RVS}

\section{A. Prelaunch RVS}

The prelaunch RVS measurements for both Terra and Aqua MODIS RSBs were performed using the SIS to illuminate the scan mirror at 13 different AOIs with three lamp illumination levels, high for bands 1, 3, 4, 8, and 9, low for bands 13-16, and medium for all other bands. Ten scans of data with each containing about 100 frames were collected during each measurement [17]. The background-subtracted instrument response is averaged over the ten center frames and five scans with the same MS for each band B and detector D. Then, the averaged background-subtracted MODIS response to the illumination of the SIS for each band, detector, and MS at 13 AOIs is fitted to a quadratic form

$$
f_{\mathrm{BDM}}(\theta)=a_{0}^{\mathrm{BDM}}+a_{2}^{\mathrm{BDM}} \theta+a_{2}^{\mathrm{BDM}} \theta^{2}
$$

where $\theta$ is the AOI. The RVS for MODIS RSB is normalized at the AOI of its onboard SD. The derived prelaunch RVS has a clear band (or wavelength) and MS dependence but a negligible detector dependence (within 0.1\%). Hence, a band-averaged prelaunch RVS was used for MODIS RSB, which was obtained using

$$
r_{\mathrm{BM}}^{\mathrm{pl}}(\theta)=\frac{1}{N_{D}} \sum_{D} f_{\mathrm{BDM}}(\theta) f_{\mathrm{BDM}}\left(\theta_{\mathrm{SD}}\right)
$$

where $N_{D}$ is the number of detector of the band and $\theta_{\mathrm{SD}}$ is the AOI of the SD.

Fig. 3 shows the prelaunch RVS for Terra bands 3, 8, and 17 . A clear dependence on the AOI and MS is seen in the prelaunch RVS of all three bands. This indicates that the two MSs of 
TABLE I

MODIS RSB KEY SPECIFICATION AND BASIC INFORMATION

\begin{tabular}{cccccccc}
\hline Band & CW & BW & IFOV & FPA & Ltyp & SNR & Primary Use \\
\hline 1 & 0.645 & 50 & 0.25 & NIR & 21.8 & 128 & Land/Cloud/Aerosols Boundaries \\
2 & 0.858 & 35 & 0.25 & NIR & 24.7 & 201 & \\
\hline 3 & 0.469 & 20 & 0.5 & VIS & 35.3 & 243 & \\
4 & 0.555 & 20 & 0.5 & VIS & 29.0 & 228 & \\
5 & 1.240 & 20 & 0.5 & SMIR & 5.4 & 74 & Land/Cloud/Aerosols Properties \\
6 & 1.640 & 24 & 0.5 & SMIR & 7.3 & 275 & \\
7 & 2.130 & 50 & 0.5 & SMIR & 1.0 & 110 & \\
\hline 8 & 0.412 & 15 & 1.0 & VIS & 44.9 & 880 & \\
9 & 0.443 & 10 & 1.0 & VIS & 41.9 & 838 & \\
10 & 0.488 & 10 & 1.0 & VIS & 32.1 & 802 & \\
11 & 0.531 & 10 & 1.0 & VIS & 27.9 & 754 & Ocean Color/Phytoplankton/ \\
12 & 0.551 & 10 & 1.0 & VIS & 21.0 & 750 & Biogeochemistry \\
13 & 0.667 & 10 & 1.0 & NIR & 9.5 & 910 & \\
14 & 0.678 & 10 & 1.0 & NIR & 8.7 & 1087 & \\
15 & 0.748 & 10 & 1.0 & NIR & 10.2 & 586 & \\
16 & 0.869 & 15 & 1.0 & NIR & 6.2 & 516 & \\
\hline 17 & 0.905 & 30 & 1.0 & NIR & 10.0 & 167 & \\
18 & 0.936 & 10 & 1.0 & NIR & 3.6 & 57 & Atmospheric Water Vapor \\
19 & 0.940 & 50 & 1.0 & NIR & 15.0 & 250 & \\
\hline 26 & 1.375 & 30 & 1.0 & SMIR & 6.0 & 150 & Cirrus Clouds Water Vapor \\
\hline & & & & & & & \\
\hline
\end{tabular}

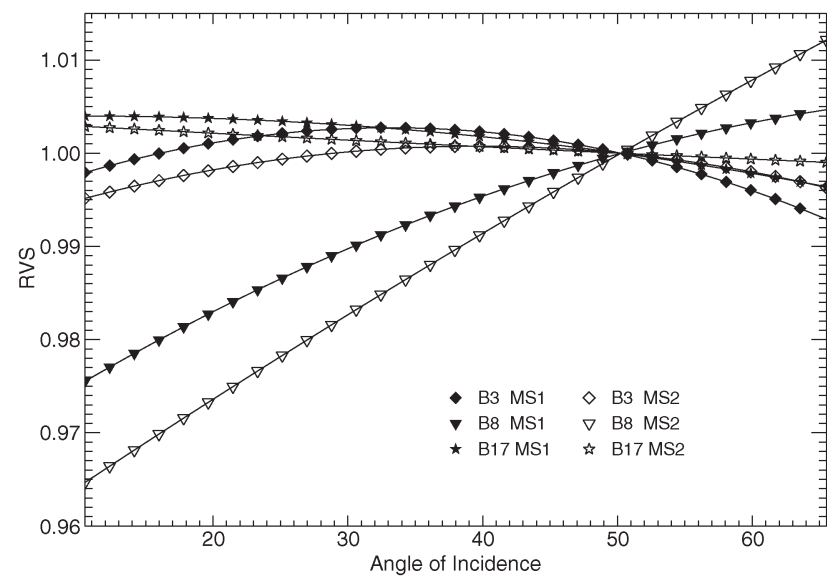

Fig. 3. Prelaunch RVS for Terra bands 3, 8, and 17.

the Terra MODIS scan mirror exhibit significantly different behavior prior to launch. For band $8(0.412 \mu \mathrm{m})$, the prelaunch RVS has a strong AOI dependence with a $5 \%$ variation over the EV scan-angle range. In the case of bands $3(0.469 \mu \mathrm{m})$ and 17 $(0.905 \mu \mathrm{m})$, the prelaunch RVS exhibits a change of less than $1 \%$ over the scan-angle range. Fig. 4 shows the prelaunch RVS for Aqua bands 3, 8, and 17. For these bands, the prelaunch RVS MS differences are less than $0.1 \%$, much smaller than those observed in Terra RSB RVS. Their AOI dependence is also less evident in comparison to that of Terra MODIS. The maximum variation of up to $1.5 \%$ is observed in the prelaunch RVS for Aqua band 8 across the entire scan-angle range. This variation is less than one-third of the corresponding behavior observed in Terra MODIS [17].

\section{B. RVS On-Orbit Update Algorithms}

The RVS of the scan mirror may change after prelaunch measurements, particularly after launch. The variations in the RVS from prelaunch characterization to the first on-orbit mea-

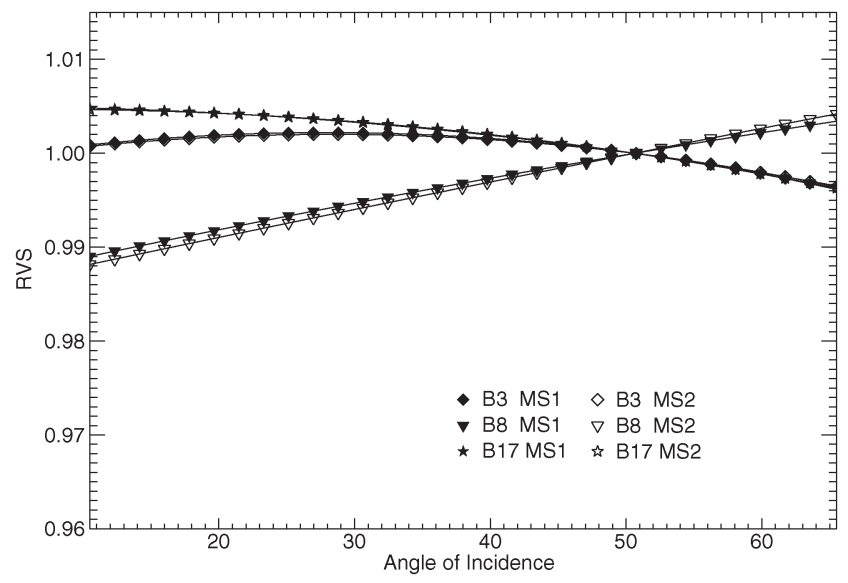

Fig. 4. Prelaunch RVS for Aqua bands 3, 8, and 17.

surements cannot be accurately characterized. Considering that the two MODIS instruments were well preserved before launch, the aforementioned variations of the RVS of the MODIS RSB are neglected in this analysis. Then, we can express the RVS as

$$
r_{\mathrm{BDM}}(\theta, t)=r_{\mathrm{BM}}^{\mathrm{pl}}(\theta) r_{\mathrm{BDM}}^{\mathrm{oo}}(\theta, t)
$$

where $r_{\mathrm{BDM}}^{\mathrm{oo}}(\theta, t)$ denotes the on-orbit RVS change for band $\mathrm{B}$, detector $\mathrm{D}$, and MS M from the time $t_{0}$ (time when the nadir door was first opened) at which $r_{\mathrm{BDM}}^{\mathrm{Oo}}(\theta, t)$ equals 1 , i.e., $r_{\mathrm{BDM}}^{\mathrm{oo}}\left(\theta, t_{0}\right)=1$. Since the RSB RVS is normalized at the AOI of the SD, the on-orbit RVS change can be expressed as

$$
r_{\mathrm{BDM}}^{\mathrm{oo}}(\theta, t)=g_{\mathrm{BDM}}(\theta, t) / g_{\mathrm{BDM}}\left(\theta_{\mathrm{SD}}, t\right)
$$

where $g_{\mathrm{BDM}}(\theta, t)$ and $g_{\mathrm{BDM}}\left(\theta_{\mathrm{SD}}, t\right)$ are the on-orbit gain change at the corresponding $\mathrm{AOI}$ and that of the $\mathrm{SD}$, respectively.

The MODIS RSB on-orbit gain change can be tracked by SD/ SDSM calibration, lunar observations, and response trending 
over selected EV sites. The knowledge of the absolute radiance of the observed objects may not be necessary for the on-orbit gain change tracking, but their reflectance must be stable during the relevant period of time. The response ratios of the two MSs at any AOI can also be obtained from the selected EV sites, which do not require long-term stability of the reflectance. The ratios can be used to derive the MS ratios of the on-orbit gain change, which enables the determination of the on-orbit RVS change for one MS from that of the other side. As the response trending over EV sites is usually noisier than that obtained from the on-orbit calibrators, there is an additional uncertainty associated with it. For most MODIS RSBs, using the onboard calibrators and the EV MS ratios is sufficient to track the onorbit RVS change and is the preferred method. However, for some RSBs, particularly the short-wavelength bands, the EV response trending is needed to accurately track the on-orbit RVS change due to either large uncertainty of the SD calibration or the necessity to track the on-orbit gain change at multiple AOIs or both. In the following sections, two approaches are presented. One is based on the measurements of the onboard calibrators and the EV MS ratios, and the other is based on the lunar and the response trending over the pseudo-invariant sites. The former is referred to as Approach I, and the latter is referred to as Approach II.

1) On-Orbit RVS Change Derivation Using Onboard Calibrators and EV MS Ratios (Approach I): As discussed earlier, the MODIS RSB gain at the AOI of the SD is tracked using the SD calibration [7]-[9]. By normalizing the gain obtained at the time that the nadir door was first opened, the on-orbit gain change at the AOI of the $\mathrm{SD}, g_{\mathrm{BDM}}\left(\theta_{\mathrm{SD}}, t\right)$, can be derived from the SD calibration. The lunar calibration that occurs approximately monthly can provide the RSB on-orbit gain change, $g_{\mathrm{BDM}}\left(\theta_{\mathrm{SV}}, t\right)$, at the AOI of the SV. Then, the RVS at the AOI of the SV can be calculated using

$$
r_{\mathrm{BDM}}^{\mathrm{oo}}\left(\theta_{\mathrm{SV}}, t\right)=g_{\mathrm{BDM}}\left(\theta_{\mathrm{SV}}, t\right) / g_{\mathrm{BDM}}\left(\theta_{\mathrm{SD}}, t\right) .
$$

Normalizing the RVS to the AOI of the SD, the on-orbit RVS change at any AOI, $r_{\mathrm{BDM}}^{\mathrm{oo}}(\theta, t)$, can be expressed using a linear approximation for the AOI dependence as follows:

$$
r_{\mathrm{BDM}}^{\mathrm{Oo}}(\theta, t)=1+\frac{\theta-\theta_{\mathrm{SD}}}{\theta_{\mathrm{SV}}-\theta_{\mathrm{SD}}}\left[r_{\mathrm{BDM}}^{\mathrm{oo}}\left(\theta_{\mathrm{SV}}, t\right)-1\right] .
$$

The instrument EV response MS ratios can be derived from ocean as well as desert sites as long as the targeted areas are spatially uniform during each observation. Compared to ocean sites, a desert target yields a higher signal-to-noise ratio (SNR) and is deemed more suitable. However, most high-gain ocean bands (bands 8-16) saturate over desert surfaces. Consequently, scenes from oceans are chosen to calculate the MS ratio [20]. Using an ocean target, the instrument response MS ratios can be obtained at multiple AOIs and can be expressed as

$$
s_{\mathrm{BD}}\left(\theta_{i}, t\right)=\frac{d n\left(B, D, M 2, \theta_{i}, t\right)}{d n\left(B, D, M 1, \theta_{i}, t\right)}, \quad i=1, \ldots, N_{1}
$$

where M1 and M2 denote the two sides and $N_{1}$ is the number of $\theta_{i}$.
With the MS ratios calculated, the RVS on-orbit variation of the two MSs can be expressed as

$$
\begin{aligned}
& r_{\mathrm{BDM} 2}^{\mathrm{oo}}\left(\theta_{i}, t\right) \\
& \quad=s_{\mathrm{BD}}\left(\theta_{i}, t\right) \frac{g_{\mathrm{BDM} 1}\left(\theta_{\mathrm{SD}}, t\right) r_{\mathrm{BM} 1}^{\mathrm{pl}}\left(\theta_{i}, t\right)}{g_{\mathrm{BDM} 2}\left(\theta_{\mathrm{SD}}, t\right) r_{\mathrm{BM} 2}^{\mathrm{pl}}\left(\theta_{i}, t\right)} r_{\mathrm{BDM} 1}^{\mathrm{oo}}\left(\theta_{i}, t\right) .
\end{aligned}
$$

Thus, if the RVS on-orbit change for M1 is known, the variation for M2 can be calculated at multiple AOIs using a suitable polynomial approximation. The linear approximation for the AOI dependence of $r_{\mathrm{BDM} 1}^{\mathrm{oo}}(\theta, t)$ may not be adequate as $r_{\mathrm{BDM} 2}^{\mathrm{Oo}}(\theta, t)$ also exhibits noticeable nonlinear dependence for most RSBs. The nonlinear behavior of the two MSs may not be identical, which may induce MS-based striping in the calibrated EV images. With (8) applied to calculate the RVS on-orbit variation for M2, the two MSs are calibrated to about the same uncertainty, and the striping effect due to the MS difference is reduced. For both Terra and Aqua MODIS RSBs, the MS ratios are calculated at ten AOIs and fitted to a quadratic form with a least-mean-square-fit approach, which is then used to derive the on-orbit RVS change for MS2 [21], [22].

2) On-Orbit RVS Change Derivation Using Desert Trending and Lunar Calibration (Approach II): Since both MODIS instruments have been operating beyond their designed lifetime of six years, there have been concerns over the accuracy of the SDbased calibration and the validity of the linear approximation for on-orbit RVS characterization, although the SD calibration and the RVS linear approximation have worked reasonably well for the first few years. To tackle these concerns, an alternative source in the form of pseudo-invariant desert sites [21] has been used to trend the MODIS RSB response. These sites are considered to be temporally stable and are endorsed by the Committee on Earth Observation Satellites (CEOS) for sensor calibration/intercomparison. In principle, one site can be used to track the MODIS RSB on-orbit change at 16 different AOIs since the MODIS orbit has 16-day repeating cycles. However, not every measurement is useful since the reflected sunlight can be impacted due to the presence of clouds above the site during observation. Therefore, multiple sites are needed to track the on-orbit gain changes at various AOIs. Three widely used sites, Libya 1 (24.42 N, 13.35 E), Libya $2(25.05 \mathrm{~N}, 20.48 \mathrm{E})$, and Libya $4(28.55 \mathrm{~N}, 23.39 \mathrm{E})$, are selected to track the RSB onorbit gain changes for both Terra and Aqua MODIS. With these three sites, the on-orbit gain change can be effectively tracked at 12 AOIs for both instruments. Since the two instruments have different orbits, the 12 AOIs are not identical, but they are close.

As mentioned earlier, lunar calibration can track the RSB on-orbit gain changes at the AOI of the SV. Because the lunar surface is very stable, the lunar calibration should be reliable and accurate. An exception is the short-wavelength infrared (SWIR) bands which have strong crosstalk among each other. For a general discussion, assume that the on-orbit gain changes are derived from the selected desert sites and lunar calibration at $N_{2}$ different AOIs and use the terms $g_{\mathrm{BM}}\left(\theta_{1}, t\right)$, $g_{\mathrm{BM}}\left(\theta_{2}, t\right), \ldots, g_{\mathrm{BM}}\left(\theta_{N_{2}}, t\right)$ to denote the on-orbit gain changes. Since the desert trending produces noisier results than that obtained from the onboard calibrators, only the detectoraveraged response is derived from the EV measurement. For 
both Terra and Aqua MODIS, $N_{2}$ is 12 or 13, depending on whether the lunar trending is included. The gains are all normalized to the time when the nadir door was first opened. At any given time $t$, there are $N_{2}$ gains at $N_{2}$ different AOIs. They can be fitted to a polynomial

$$
h_{\mathrm{BM}}(\theta, t)=\sum_{i=0}^{P} b_{i}(B, M, t) \theta^{i}
$$

where $P$ is the order of the polynomial. If the lunar measurement is used in the fitting, the polynomial is constrained to pass through the lunar response trending.

Using the fitted polynomial, a detector-averaged RVS onorbit variation can be derived using

$$
r_{\mathrm{BM}}^{\mathrm{oo}}(\theta, t)=\frac{h_{\mathrm{BM}}(\theta, t)}{h_{\mathrm{BM}}\left(\theta_{\mathrm{SD}}, t\right)}
$$

where the normalization factor $h_{\mathrm{BM}}\left(\theta_{\mathrm{SD}}, t\right)$ provides the onorbit gain change at the AOI of the SD. The RVS on-orbit detector-dependent differences can be derived from the SD and lunar calibration with a linear approximation for the AOI dependence.

\section{RVS LUTS}

MODIS bands 5-7 and 26 are SWIR bands. No evidence has demonstrated that the RVS for these bands has noticeable change for both instruments since launch. Thus, prelaunch RVS has been applied to them in all MODIS L1B versions. For Terra bands 10-19 and Aqua bands 1-4 and 10-19, SD and lunar calibration sufficiently track the orbit gain change at the AOI of the SD and that of the SV, and the linear approximation for the AOI dependence of the on-orbit RVS change works well. For these bands, the RVS on-orbit changes are derived using Approach I with the MS 1 on-orbit RVS change calculated using (6) and that for MS 2 calculated using (8). In principle, (6) can be applied to either MSs. However, there was a "smoking" event for Terra MODIS during the prelaunch measurements [8], which provides some justification for the aforementioned choice for Terra MODIS. To be consistent, the same choice is applied to Aqua MODIS [22], [23].

For Terra MODIS bands 1-4, 8, and 9 and Aqua MODIS bands 8 and 9 , the response trending over the pseudo-invariant desert sites reveals that the linearity assumption about the AOI dependence of the RVS on-orbit variation does not hold [2]. Comparison between the desert response trending around the AOI of the SD and the SD response trending also reveals that the SD measurements cannot reliably capture the on-orbit gain change for Terra bands 1-4, 8, and 9, particularly for the past few years [22]. For the aforementioned bands, Approach II is applied to derive the on-orbit RVS change. For these bands, the lunar response trending matches the desert response trending at the SV AOI quite well, and a quartic form is applied to describe the AOI dependence except for Terra bands 1 and 2. For these two bands, the lunar response trending does not match the desert response trending at the SV AOI, and only the response trending over desert sites is used to derive the on-orbit change with the same quadratic form being applied to describe the AOI dependence. The exact reason why the lunar trending does not match the desert trending at the SV AOI for Terra bands 1-2 is still unknown.

With the prelaunch RVS $r_{\mathrm{BM}}^{\mathrm{pl}}(\theta)$ and the RVS on-orbit variation $r_{\mathrm{BDM}}^{\mathrm{oo}}(\theta, t)$, the time-dependent $\operatorname{RVS} r_{\mathrm{BDM}}(\theta, t)$ can be easily calculated for each band, MS, detector, and AOI. The EV frame number $F$, ranging from 0 to 1353 , and the AOI $\theta$ are related by

$$
\theta=\frac{65.5^{\circ}-10.5^{\circ}}{1353} F+10.5^{\circ}
$$

For MODIS L1B, the RVS is refitted as a polynomial function of the EV frame number

$$
r_{\mathrm{BDM}}^{\mathrm{L} 1 \mathrm{~B}}(F, t)=\sum_{i=0}^{Q} c_{i}(B, M, t) F^{i}
$$

where $Q$ is the order of the polynomial and is band dependent. For Terra MODIS bands 3, 4, 8, and 9 and Aqua bands 8 and 9, $Q$ is 4, while for all other bands, it is 2 . The fitted coefficients of all the RSBs and the two MSs form a MODIS RSB RVS lookup table (LUT) with a time stamp $t$. The LUT is generated at a set of selected time stamps and used in MODIS L1B as input.

Table II is a summary of MODIS RSB RVS update algorithms. The first column in the table is the band number. The second and sixth columns provide the methods used to derive the RVS: Prl denotes prelaunch RVS, while App. I and App. II indicate an RVS update using Approach I and Approach II, respectively. The third and seventh columns indicate the order of the polynomial used to describe the AOI dependence of the RVS on-orbit change at any given time. The fourth and eighth columns are the polynomial order used to describe the AOI dependence of the time-dependent RVS at any specified time. The fifth and tenth columns provide the information for the data used to update the RVS on orbit: DT for pseudoinvariant desert data, Moon for lunar data, SD for SD data, and OM for MS ratios obtained from ocean sites.

\section{ON-ORBIT GAIN ChAngES}

\section{A. On-Orbit Gain Change From Onboard Calibrators and EV MS Ratios}

1) SD/SDSM Calibration: During the first year on orbit, both Terra and Aqua MODIS performed the SD calibration on a weekly basis. The frequency of SD calibration was reduced to biweekly after the first year. Since July 2, 2003, the SD door of Terra MODIS has been kept in the "open" position, and the SD screen is in place on a permanent basis. Since the SD door is always open, an SD calibration is performed every orbit. Prior to July 2, 2003, the SDSM was operated at the same frequency as the SD, and thereafter, it is operated once every three weeks. In the case of Aqua MODIS, the SD/SDSM scheduling frequency was further reduced to once every three weeks to prolong the lifetime of the SD/SDSM calibration capability. With the onboard SD/SDSM calibration, the gain of the MODIS electronic and optical system at the AOI of the $\mathrm{SD}$ can be derived. For each calibration event at time $t$, the 
TABLE II

Information for MODis RSB Time-Dependent RVS. DT: Desert Targets. Prl: Prelaunch. OM: Ocean Site MS Ratios

\begin{tabular}{|c|c|c|c|c|c|c|c|c|}
\hline \multirow{2}{*}{ Band } & \multicolumn{9}{|c|}{ Terra } & \multicolumn{3}{|c|}{ Aqua } \\
\cline { 2 - 9 } & App. & P & Q & Data & App. & P & Q & Data \\
\hline 1 & App. II & 2 & 2 & DT & App. I & 2 & 2 & SD, Moon, OM \\
\hline 2 & App. II & 2 & 2 & DT & App. I & 2 & 2 & SD, Moon, OM \\
\hline 3 & App. II & 4 & 4 & DT, Moon & App. I & 4 & 4 & SD, Moon, OM \\
\hline 4 & App. II & 4 & 4 & DT, Moon & App. I & 4 & 4 & SD, Moon, OM \\
\hline 5 & PrI & 0 & 2 & & Prl & 0 & 2 & \\
\hline 6 & Prl & 0 & 2 & & Prl & 0 & 2 & \\
\hline 7 & Prl & 0 & 2 & & Prl & 0 & 2 & \\
\hline 8 & App. II & 4 & 4 & DT, Moon & App. II & 4 & 4 & DT, Moon \\
\hline 9 & App. II & 4 & 4 & DT, Moon & App. II & 4 & 4 & DT, Moon \\
\hline 10 & App. I & 1 & 2 & SD, Moon, OM & App. I & 1 & 2 & SD, Moon, OM \\
\hline 11 & App. I & 1 & 2 & SD, Moon, OM & App. I & 1 & 2 & SD, Moon, OM \\
\hline 12 & App. I & 1 & 2 & SD, Moon, OM & App. I & 1 & 2 & SD, Moon, OM \\
\hline 13 & App. I & 1 & 2 & SD, Moon, OM & App. I & 1 & 2 & SD, Moon, OM \\
\hline 14 & App. I & 1 & 2 & SD, Moon, OM & App. I & 1 & 2 & SD, Moon, OM \\
\hline 15 & App. I & 1 & 2 & SD, Moon, OM & App. I & 1 & 2 & SD, Moon, OM \\
\hline 16 & App. I & 1 & 2 & SD, Moon, OM & App. I & 1 & 2 & SD, Moon, OM \\
\hline 17 & App. I & 1 & 2 & SD, Moon & App. I & 1 & 2 & SD, Moon \\
\hline 18 & App. I & 1 & 2 & SD, Moon & App. I & 1 & 2 & SD, Moon \\
\hline 19 & App. I & 1 & 2 & SD, Moon & App. I & 1 & 2 & SD, Moon \\
\hline 26 & Prl & 0 & 2 & & Prl & 0 & 2 & \\
\hline
\end{tabular}

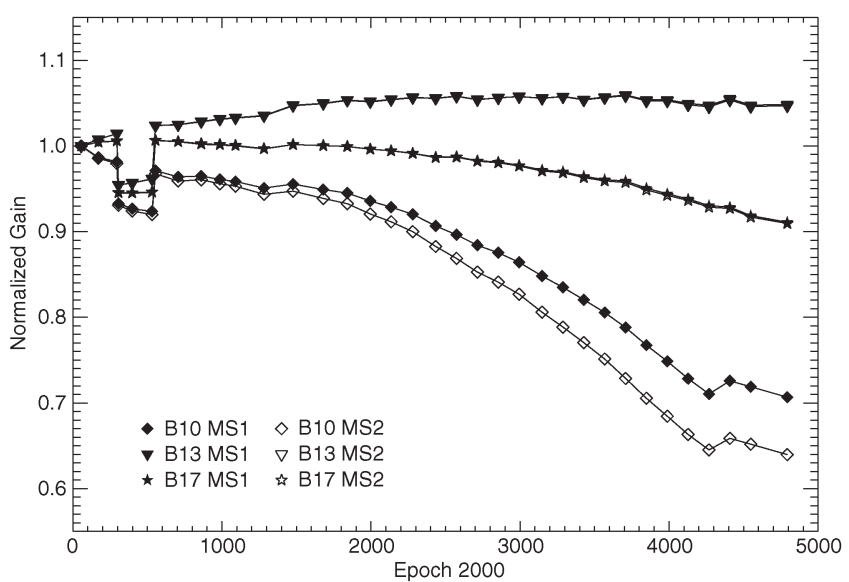

Fig. 5. Gain trending for Terra MODIS bands 10, 13, and 17, derived from the SD calibration.

calibration coefficient denoted as $\mathrm{m}_{1}$ in this paper and in the literature [7]-[9] is derived for each band, detector, subframe, and MS. The gain at the AOI of the SD is inversely proportional to the calibration coefficient, and then the on-orbit gain change can be calculated by inversing the calibration coefficient.

Fig. 5 shows the SD gain trending for the selected RSBs, which have been averaged over detectors and normalized to the time when the nadir door first opened. The filled symbols denote the data for MS 1 while unfilled symbols are for MS 2. The discontinuities around day 305 (October 31, 2000) and day 549 (July 2, 2001) are due to the changes of the MODIS electronic configuration from side A to side B and back to side A from side B, respectively. The increased rate of degradation beyond day 1279 (July 2, 2003) is due to an increased exposure to sunlight

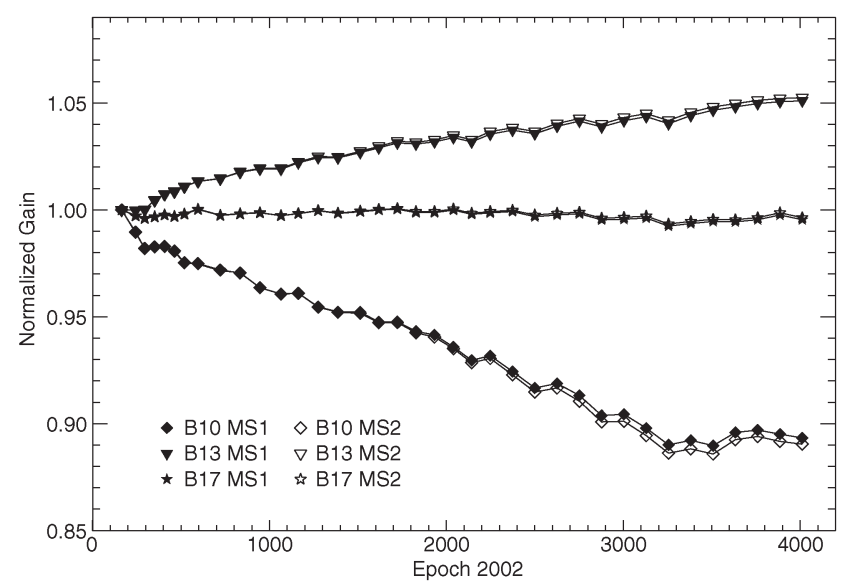

Fig. 6. Gain trending for Aqua MODIS bands 10, 13, and 17, derived from the SD calibration.

after the SD door for Terra MODIS was permanently left in the open position. Even though the on-orbit change in the SD is characterized using the SDSM, the increased degradation rate of the optics, including the scan mirror, results in a larger gain change after day 1279. The gain trending for band $10(0.488$ $\mu \mathrm{m})$ shows more change compared to the longer wavelength band $17(0.905 \mu \mathrm{m})$, while band $13(0.667 \mu \mathrm{m})$ shows a gain increase over the mission. Among the bands shown in Fig. 5, band $10(0.488 \mu \mathrm{m})$ has the shortest wavelength and shows the most change, about 30\% for MS 1 and 36\% for MS 2 .

The Aqua MODIS RSB gain derived from the SD is shown in Fig. 6 for bands $10(0.488 \mu \mathrm{m}), 13(0.667 \mu \mathrm{m})$, and 17 $(0.905 \mu \mathrm{m})$. Similar to Fig. 5, the gain coefficients have been averaged over detectors, fitted to piecewise smooth functions, 


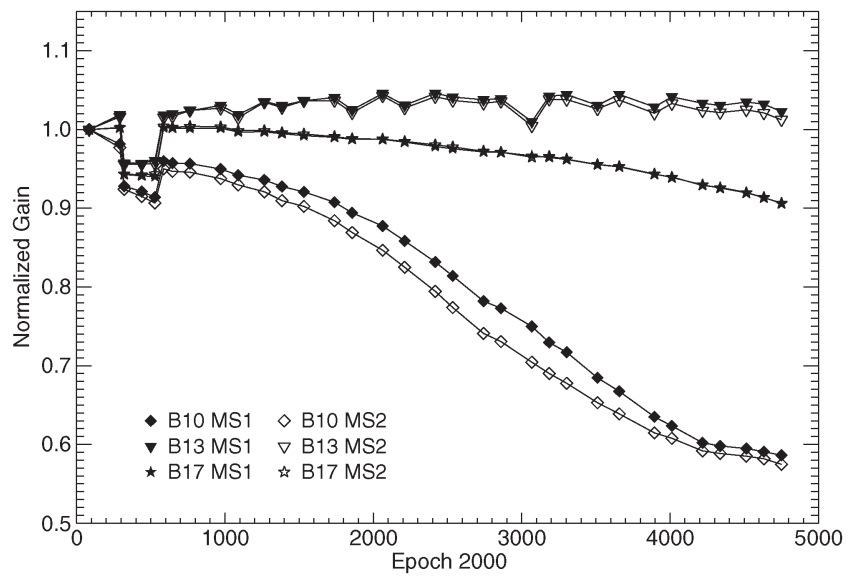

Fig. 7. Gain trending for Terra MODIS bands 10, 13, and 17, derived from the lunar calibration.

and normalized to the time when the nadir door was first opened. Similar to the Terra band $13(0.667 \mu \mathrm{m})$ trending shown in Fig. 5, the gain of Aqua band $13(0.667 \mu \mathrm{m})$ derived from SD calibration also increases over time. The largest gain change among the three bands is seen for band $10(0.488 \mu \mathrm{m})$, consistent with the trend observed in Terra MODIS. The gain for Aqua band $10(0.488 \mu \mathrm{m})$ shows a decrease of about $11 \%$ which is of a smaller magnitude in comparison to Terra band $10(0.488 \mu \mathrm{m})$. The MS differences in the gain trending are also much smaller than those observed in Terra RSB.

2) Lunar Calibration: Both Terra and Aqua MODIS have been scheduled to view the Moon approximately monthly, about nine months every year, through their SV [18], [19]. The lunar irradiance depends on lunar view geometry. To reduce the uncertainty of the view geometry effect correction, the lunar phase angle, defined as negative for a waxing moon and positive for a waning moon, is usually kept in the range $\left[-56^{\circ},-55^{\circ}\right]$ for Aqua MODIS and in the range $\left[55^{\circ}, 56^{\circ}\right]$ for Terra MODIS, respectively. Lunar calibration can be used to track the on-orbit gain change at the AOI of the SV as long as the data are not saturated during a lunar observation for each band, detector, and MS.

The detector-averaged on-orbit gain changes derived from lunar calibration for Terra MODIS bands 10, 13, and 17 are shown in Fig. 7. Similar to Fig. 5, the discontinuities around day 305 (October 31, 2000) and day 549 (July 2, 2001) are due to the previously mentioned changes of the MODIS electronic configuration. Gain degradation rate changes around day 1279 are due to the SD door remaining open starting on July 2, 2003. A similar wavelength-dependent degradation pattern is observed from the lunar gains. Band $10(0.488 \mu \mathrm{m})$ shows a degradation of about $41.5 \%$ for MS 1 and $42.5 \%$ for MS 2 at the AOI of the SV, which is about $11.5 \%$ and $6.5 \%$ more that those at the AOI of the SD as demonstrated in Fig. 5 for MSs 1 and 2, respectively. Differences between the SD and lunar trending are also seen for other bands, particularly for shortwavelength bands, and justify the need for an on-orbit RVS characterization.

The Aqua MODIS RSB detector-averaged gain derived from lunar calibration is shown in Fig. 8 for bands 10, 13, and 17.

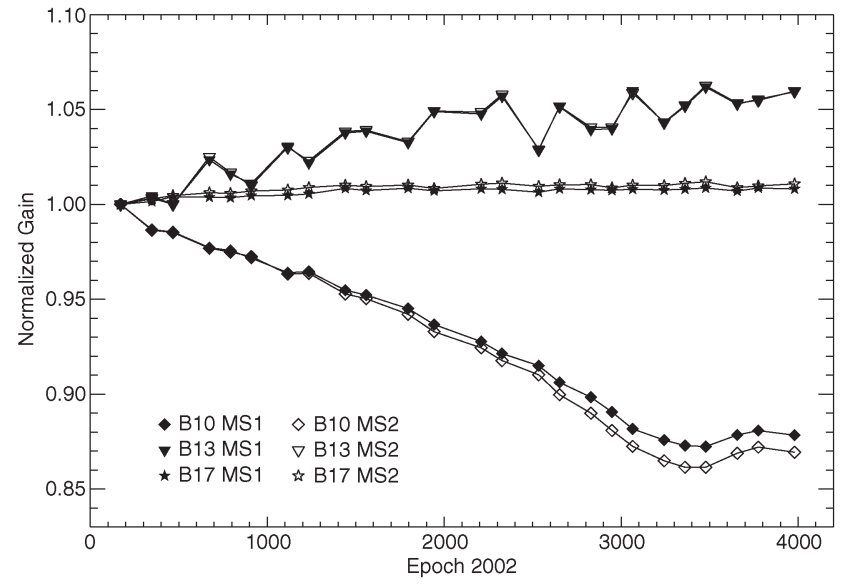

Fig. 8. Gain trending for Aqua MODIS bands 10, 13, and 17, derived from the lunar calibration.

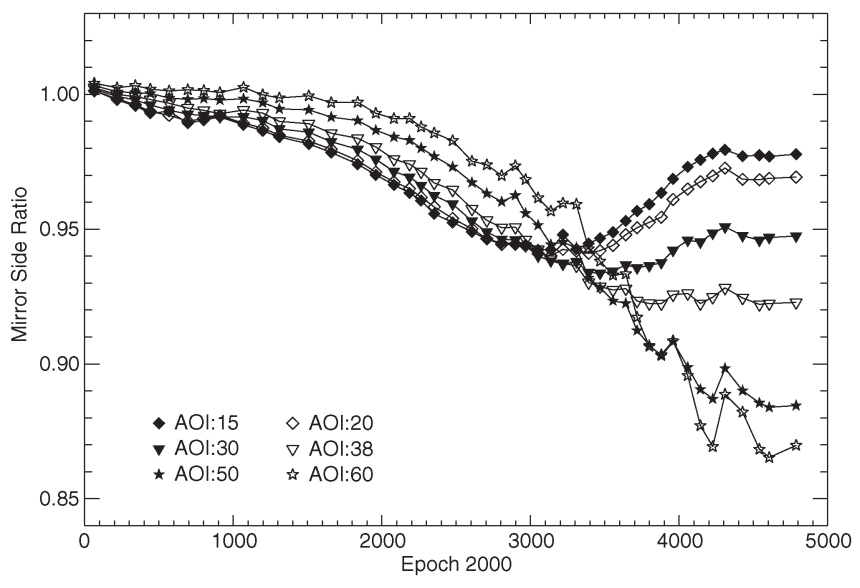

Fig. 9. Terra band 10 EV MS ratio trending observed at ocean sites in Pacific Ocean around the equator.

Similar to the Terra results, the gain exhibits wavelength dependence since band $13(0.667 \mu \mathrm{m})$ is saturated while viewing the Moon. Hence, a ratio approach using an unsaturated band as reference is applied to derive the lunar response trending [20], which may increase the uncertainty for the derived gains seen in Fig. 8. For band $10(0.488 \mu \mathrm{m})$, the gain at the AOI of the SV decreases by about $12 \%$ and $13 \%$ for MSs 1 and 2, respectively. This is about $1 \%$ and $2 \%$ more than the corresponding results using the SD calibration method. Compared to Terra band 10 $(0.488 \mu \mathrm{m})$, the gain change as well as the difference between the two sources is much smaller.

3) MS Ratios Over Ocean Sites: Most MODIS high-gain ocean color bands (8-16) saturate while observing land targets. Hence, ocean sites are selected to track the MODIS RSB MS ratios. In addition, a uniform ocean scene can provide measurements at multiple AOIs within a single granule. To reduce the seasonal oscillation induced by the polarization effect, the equator area in the Pacific Ocean with a longitude of $-136^{\circ}$ was used to obtain the response ratios used in calculation of the MODIS C6 LUT. From an EV granule which covers the equator area in the Pacific Ocean, the MS ratios are calculated at selected AOIs.

Fig. 9 shows the trending of the EV MS ratios for Terra MODIS band $10(0.48 \mu \mathrm{m})$ at six specific AOI. It is clearly 


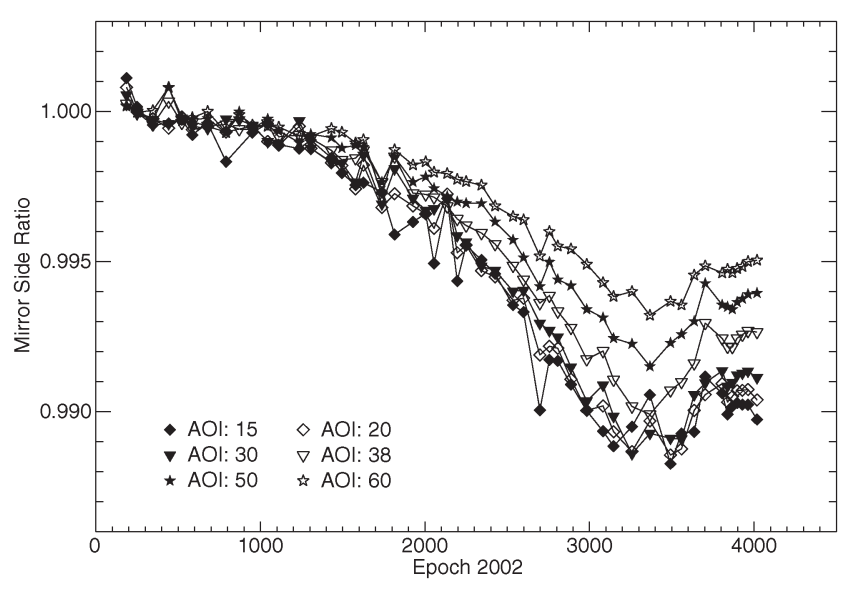

Fig. 10. Aqua band 10 EV MS ratio trending observed at ocean sites in Pacific Ocean around the equator.

seen that the MS ratios change with time and are strongly AOI dependent. A seasonal oscillation in recent years is also seen in the MS ratios at large AOI. This yearly oscillation in recent years (since 2007) is primarily due to the fact that MODIS is a polarization-sensitive instrument and the sensitivity is MS and time dependent, particularly at large AOI [27]. Fig. 10 shows the corresponding Aqua band $10 \mathrm{MS}$ ratio trending at the same six AOIs. In comparison with Terra band 10, Aqua band 10 exhibits a much more stable MS response ratio.

\section{B. On-Orbit Gain Change From Pseudo-invariant Desert Sites}

1) Response Trending Over Desert Sites: The EV responses at different AOIs are derived from the noncalibrated Level 1A (L1A) covering the selected pseudoinvariant desert targets. The EV responses are averaged over a $20 \times 20 \mathrm{~km}$ area centered at each site. The footprint of the selected $20 \times 20 \mathrm{~km}$ region at $1-\mathrm{km}$ nadir resolution covers one scan for each MS with ten along-track detectors and 20 frames in each scan. The data from high-resolution bands (bands 1-7) are aggregated to the 1-km resolution. The L1A granules with a 16-day repeat cycle are used to group the collected EV responses from a given site, so the responses in each group have a roughly identical AOI over the entire mission.

The EV data are corrected for the background signal, the instrument temperature effects, the cosine effect of the incident sunlight, and the Earth-Sun distance variation over the orbits. The saturated pixels contaminated due to clouds are excluded. A uniformity filter using the combined standard errors of the reflectance of one VIS band (band 1, $0.645 \mu \mathrm{m}$ ) and one NIR band (band 2, $0.858 \mu \mathrm{m}$ ) is also applied to remove the cloudcontaminated data. All remaining EV pixels after the uniformity filtering are then averaged over detectors and 20 frames in each scan to derive the MS-dependent EV response.

Fig. 11 shows the derived EV response trending at three different scan angles (AOI) for Terra MODIS band $8(0.412 \mu \mathrm{m})$ MS 1. The yearly oscillation in the EV response at frame 111 is largely the effect of the test site bidirectional reflectance distribution function (BRDF), which is dependent on the seasonal solar viewing geometry changes. The large slope and discontinuity in the early part of the mission is due to the

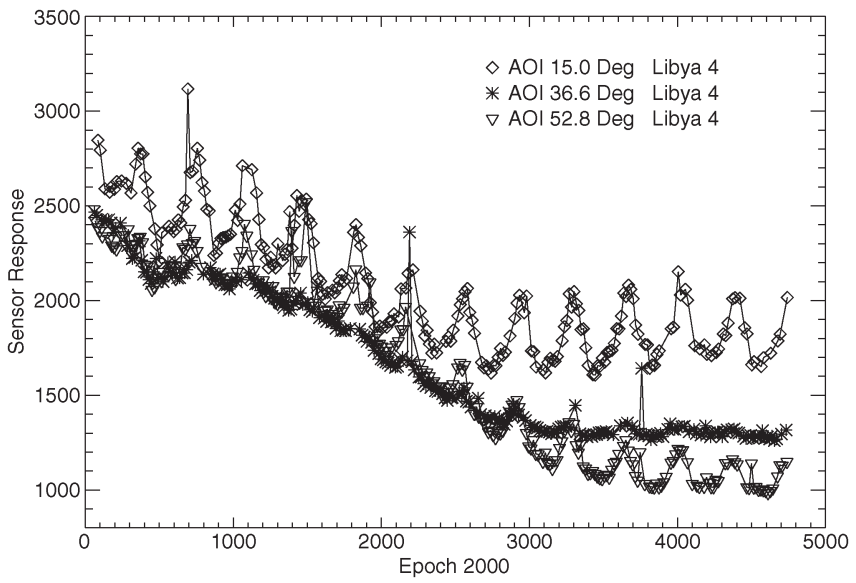

Fig. 11. EV response for Terra MODIS band 8 MS 1 over the pseudoinvariant desert sites without BRDF correction.

change in the electronic configuration for Terra MODIS. The discontinuity will be eliminated after the application of the calibration coefficients. A smaller yearly oscillation is observed in the trending at the AOI of $36.6^{\circ}$, also affected by the site BRDF. There is seasonal oscillation in the trending for the AOI of $52.8^{\circ}$ as well, with the amplitude of oscillation greatly increasing after 2008. The amplitude increase is due to the polarization property change of the band. In order to have a better assessment of the actual instrument gain change, the impacts of these yearly oscillations must be mitigated before further processing.

A semiempirical BRDF model developed by Roujean et al. [25] is chosen to perform the BRDF normalization. The kerneldriven BRDF model is expressed as follows:

$\rho(\lambda, \theta, \varphi, \psi)=k_{0}(\lambda)+k_{1}(\lambda) f_{1}(\theta, \varphi, \psi)+k_{2}(\lambda) f_{2}(\theta, \varphi, \psi)$

where $\lambda$ is the band wavelength, $\theta, \varphi$, and $\psi$ are the solar zenith angle, sensor zenith angle, and relative azimuth angle, respectively, and $\rho$ is the modeled top-of-atmospheric (TOA) reflectance. The kernel $f_{1}$ accounts for the volume scattering, and the kernel $f_{2}$ represents the surface scattering and geometric shadow casting. The site-specific BRDF coefficients $k_{0}, k_{1}$, and $k_{2}$ quantify the contributions to the TOA reflectance by combining the isotropic component with the two kernels describing anisotropic components. They are related to the physical structure and optical properties of the EV target site. The site- and AOI-specific BRDF coefficients can be derived from the linear regression to the observed MODIS TOA reflectance. The onboard calibration is known to be able to provide stable trends for the L1B product (i.e., radiance or reflectance) during the initial years after launch. Therefore, the observed TOA reflectance derived using the onboard calibrations in the first three years of the mission for both Terra and Aqua MODIS is used to determine the BRDF coefficients at each selected desert site and AOI. Since uncertainties are significantly larger to derive the coefficients that can cover the entire range of the MODIS scan angle due to the impact of the underlying atmosphere, the BRDF coefficients are actually derived for each individually fixed AOI using the corresponding EV data. 


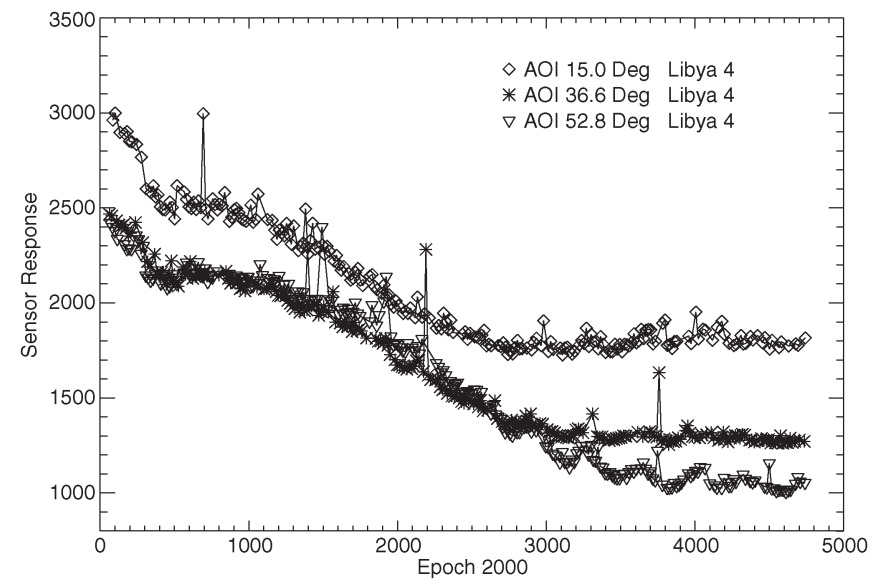

Fig. 12. EV response for Terra MODIS band 8 MS 1 over the pseudoinvariant desert sites after BRDF correction.

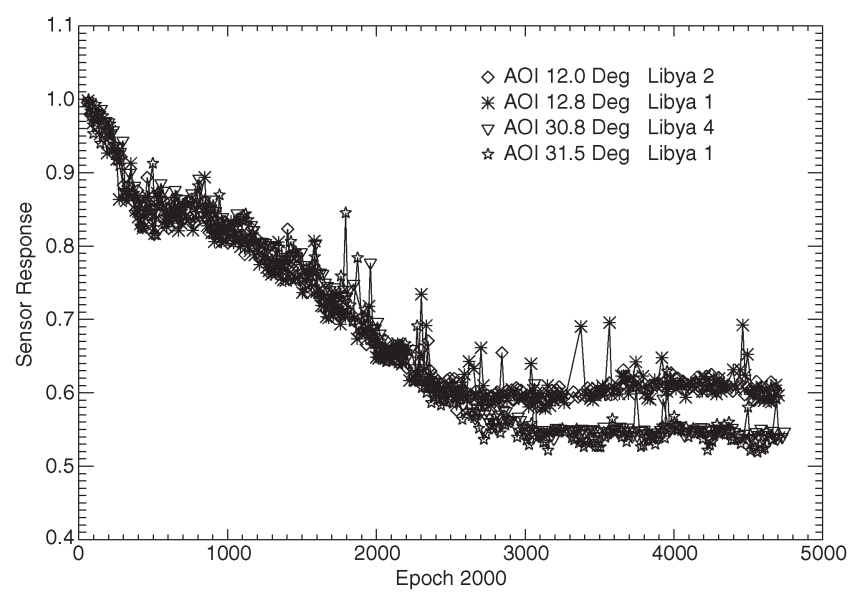

Fig. 13. EV response for Band $8 \mathrm{MS} 1$ at multiple sites for each AOI.

Fig. 12 shows the Terra MODIS band 8 MS 1 EV response trending after the BRDF correction. Significant improvement in reducing the yearly oscillation is seen when compared with the EV responses in Fig. 11. The seasonal variation may still be seen at large frame numbers after 2008 for Terra short-wave bands, as shown in Fig. 12 for band 8. The effect is primarily due to the polarization property change of the MODIS scan mirror [26], [27].

2) Comparison Among Different Sites and With the Lunar Observations: The radiometric stability of the selected sites needs to be investigated before the instrument responses measured at the sites are applied to track the MODIS RSB onorbit gain change. Fig. 13 shows the Terra band $8(0.412 \mu \mathrm{m})$ responses observed at sites Libya $1\left(24.42^{\circ}, 13.35^{\circ}\right)$, Libya 2 $\left(25.05^{\circ}, 20.48^{\circ}\right)$, Libya $4\left(28.55^{\circ}, 23.39^{\circ}\right)$, and Mali $1\left(19.12^{\circ}\right.$, $-4.85^{\circ}$ ) for AOIs of $12.0^{\circ}, 12.8^{\circ}, 30.8^{\circ}$, and $31.5^{\circ}$. Due to the wide MODIS swath and the proximity of the location of these desert sites, it is difficult to obtain the response from two different sites at the exact same AOI. In this case, the responses from AOIs of $12.0^{\circ}$ and $30.8^{\circ}$ are compared with the responses derived from AOIs of $12.8^{\circ}$ and $31.5^{\circ}$, respectively. The responses of the first three years have been fitted to a smooth function of time and then normalized to the time when

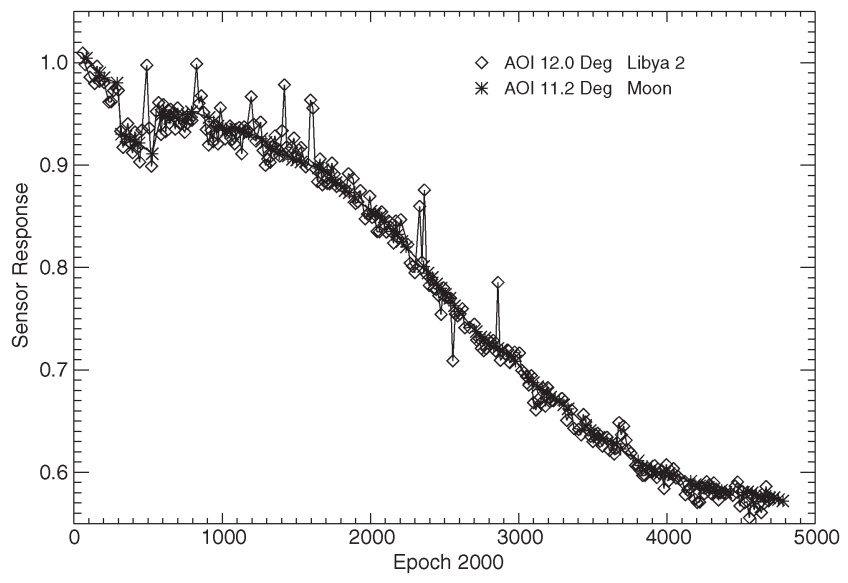

Fig. 14. EV and lunar response for Terra MODIS band 8 MS 1.

the nadir door was first opened. The first sets of data at AOIs of $12.0^{\circ}$ and $12.8^{\circ}$ provide similar response trending results for Terra band $8(0.412 \mu \mathrm{m})$ at the AOI of $12.4^{\circ}$. The long-term difference between the two trending results is less than $1 \%$. The last sets of data at AOIs of $30.8^{\circ}$ and $31.5^{\circ}$ provide the response trending results for Terra band $8(0.412 \mu \mathrm{m})$ at the AOI of $31.1^{\circ}$. Once again, the difference between the two trending results is less than $1 \%$. Considering the uncertainty of the EV data and the small difference of the AOI, the two sets of trending results agree reasonably well. A similar conclusion can be obtained for Terra bands 1-4, 8, and 9 and Aqua bands 8 and 9, which also use the instrument responses over the desert sites to track the gain on-orbit changes at the selected AOI.

It is well known that the Moon surface reflectance is very stable in the VIS and NIR band range. Thus, another direct validation of the radiometric stability of the desert sites is to compare the response trending observed at the desert sites with that obtained from the lunar observations. Fig. 14 shows the response change from the lunar view and desert site observation at the AOI of $12.0^{\circ}$ which is very close to $11.2^{\circ}$, the AOI of the $\mathrm{SV}$ or the lunar view. The symbols are for measured data. The data are normalized at the time that the nadir first opened by applying the fitted smooth function for each of the observations. From Fig. 14, it can be seen that the response changes observed from the lunar view and desert site agree very well (within 1\%). It is a direct validation for using the response change observed from the desert sites for tracking the on-orbit gain change for these bands. A similar trend is observed for most RSBs with the exception of the high-gain ocean bands and Terra bands 1 and 2. For the high-gain ocean bands, they are saturated when they view the desert sites, and hence their on-orbit gain variation change cannot be tracked. In the case of Terra bands 1 and 2, the EV and lunar response changes show a measurable disagreement in their responses. However, a contrary result is observed in the case of Aqua MODIS where a reasonable agreement (within $0.5 \%$ ) is observed between the EV and lunar measurements. The cause for the difference between the lunar and EV responses of Terra bands 1 and 2 remains unknown. Due to the disagreement between the lunar and EV response trending, the lunar data are not used in the derivation of the onorbit RVS change for these two bands. 


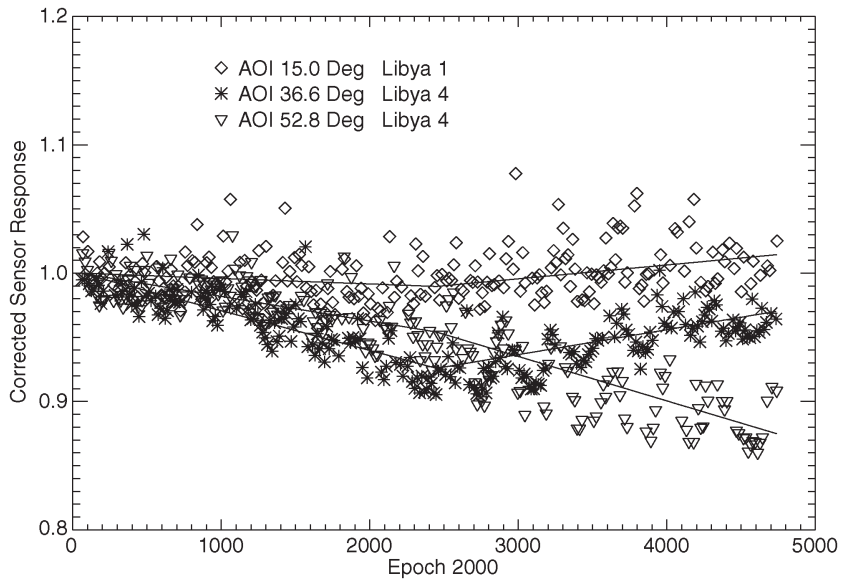

Fig. 15. Corrected EV response for Terra MODIS band 8 MS 1 along with the two-piece fitting model.

3) Measured Gain Fitted to Smooth Functions: The rate of the RSB gain change varies as a function of AOI, time, wavelength, and MS. Several smoothly connected functions have to be used to describe the SD and lunar on-orbit gain variation for each RSB. The EV response trending post-BRDF normalization has a large uncertainty, and the direct fitting of these data to piecewise smooth functions may prove inaccurate in describing the EV response. In order to avoid the larger uncertainties in the LUT, an alternative way to describe the EV response trending is described using via the function:

$g_{\mathrm{BM}}^{0}(\theta, t)=\frac{\left(50.25^{\circ}-\theta\right)}{50.25^{\circ}-11.2^{\circ}} g_{\mathrm{BM}}^{\mathrm{Moon}}(t)+\frac{\left(\theta-11.2^{\circ}\right)}{50.25^{\circ}-11.2^{\circ}} g_{\mathrm{BM}}^{\mathrm{SD}}(t)$

where $g_{\mathrm{BM}}^{\mathrm{Moon}}(t)$ and $g_{\mathrm{BM}}^{\mathrm{SD}}(t)$ are the fitted smooth functions for lunar and SD gains normalized at the time $t_{0}$ of the first opening of the nadir door. The values $50.25^{\circ}$ and $11.25^{\circ}$ correspond to the SD AOI and lunar AOI, respectively. $g_{\mathrm{BM}}^{0}(\theta, t)$ denotes the calibration coefficients derived from the SD calibration over Approach I RVS ( $\left.\mathrm{m}_{1} / \mathrm{RVS}\right)$ on-orbit change. Then, a corrected $\mathrm{EV}$ response can be defined

$$
d n_{\mathrm{EV}}^{\prime \prime}\left(B, M, \theta_{i}, t\right)=d n_{\mathrm{EV}}^{\prime}\left(B, M, \theta_{i}, t\right) / g_{\mathrm{BM}}^{0}\left(\theta_{i}, t\right)
$$

for band $\mathrm{B}$ and MS M at AOI $\theta_{i}$, which has the same long-term drift as the EV reflectance if the SD calibration coefficients and Approach I RVS are applied in MODIS L1B. Fig. 15 shows the normalized $d n_{\mathrm{EV}}^{\prime \prime}$ trending for Terra MODIS band 8 $(0.412 \mu \mathrm{m})$ at three AOIs, which are close to SV, nadir, and SD AOI. Compared to the Terra band $8 \mathrm{EV}$ response trending in Fig. 12, the $d n_{\mathrm{EV}}^{\prime \prime}$ trending has a much simpler dependence of time and can be well described by a two-piece linear function, which is also used to fit the normalized $d n_{\mathrm{EV}}^{\prime \prime}$ trending for Terra bands 3 and 9. In the case of Terra bands 1, 2, and 4, the corrected EV response is described using a one-piece linear function, whereas a one-piece quartic model is applied for Aqua MODIS. If $g_{\mathrm{BM}}^{1}\left(\theta_{i}, t\right)$ represents the fitted smooth function for band $\mathrm{B}$ and MS M at AOI $\theta_{i}$, then the gain on-orbit change at AOI $\theta_{i}$ derived from $\mathrm{EV}$ response can be expressed as

$$
g_{\mathrm{BM}}\left(\theta_{i}, t\right)=g_{\mathrm{BM}}^{0}\left(\theta_{i}, t\right) g_{\mathrm{BM}}^{1}\left(\theta_{i}, t\right)
$$

Each MODIS can view a selected desert site at multiple AOIs due to the 16-day orbit repeat cycle. Because the AOIs of different sites are usually slightly different due to their geolocation differences, a set of approximately evenly distributed AOIs for each instrument can be selected to track the on-orbit gain changes.

\section{RVS AND ITS ON-ORBIT PERFORMANCE}

\section{A. On-Orbit Time-Dependent RVS}

For Terra bands 10-19 and Aqua bands 1-4 and 10-19, the RVS on-orbit change is derived using the SD/SDSM and lunar calibrations with a linear assumption for the AOI dependence (Approach I). For Terra bands 1-4, 8, and 9 and Aqua bands 8 and 9, the RVS on-orbit change is derived from the EV response trending over pseudo-invariant desert sites at selected $\mathrm{AOI}$ and lunar response trending with higher order polynomial approximation for the AOI dependence (Approach II). The response trending is obtained either from the onboard calibrators or desert sites, fitted to a piecewise smooth connected function, and normalized to the time that the nadir door first opened for each AOI, band, and MS. At any given time, the normalized response is used to describe the behavior of AOI dependence using a polynomial approximation. Then, the derived polynomial is normalized at the AOI of the SD. This normalized polynomial is the on-orbit RVS change at the time since the nadir door opened.

The inverse of the normalization factor provides the on-orbit change of the calibration coefficient $m_{1}$. For the Approach I bands, the on-orbit gain change at the AOI of the SD is also provided by the SD/SDSM calibration, and thus, the inverse of the normalization factor matches the on-orbit change of the calibration coefficient derived from the SD/SDSM calibration [7]-[9]. For bands calibrated with Approach II, the on-orbit gain changes are mainly tracked by the response trending over the pseudoinvariant desert sites, and the on-orbit change of the calibration coefficient provided by the normalization factor does not necessarily match that derived from the SD/SDSM calibration. The difference between them shows the inadequacy and uncertainty of the SD/SDSM calibration.

Fig. 16 shows the RVS on-orbit change for Terra Band 8 $(0.412 \mu \mathrm{m})$ as a function of AOI at particular time stamps. It is clearly seen that a quartic form is needed to accurately describe the AOI dependence of the on-orbit RVS change. The solid symbols denote the on-orbit lunar measurements which also exhibit a good agreement with the EV response around the same AOI. At any given time, the factor used to normalize each fitted polynomial is the degradation of the gain at the AOI of the $\mathrm{SD}$. Hence, the gain at the AOI of the SD is also determined in addition to the RVS using the lunar and EV response trending. The relative RVS curve shows the most temporal change at the AOI of the SV. This is also true for other bands using a quartic form to describe the AOI dependence. For Terra bands $1-2$, the lunar view response trending data do not match the EV response trending results and hence are not included in the RVS derivation for these bands as mentioned in Section II. For these two bands, a quadratic form is sufficient to fit the EV response trending results at the selected AOI for any given time. 


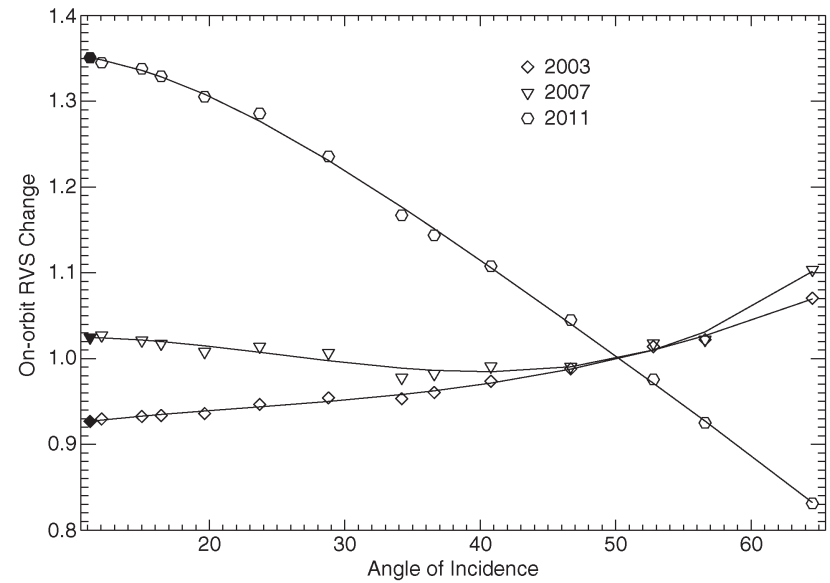

Fig. 16. Relative RVS for Terra MODIS band 8 MS 1.

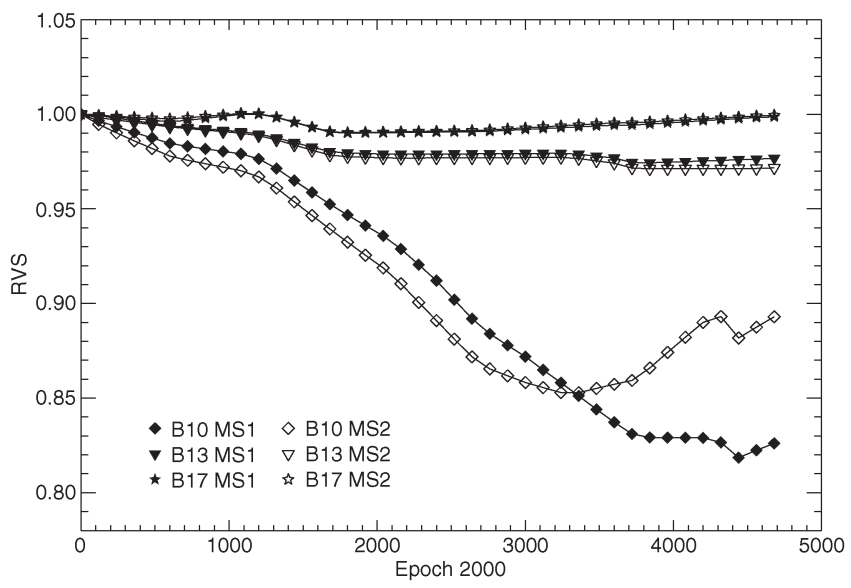

Fig. 17. RVS for Terra MODIS bands 10, 13, and 17 at the AOI of the SV.

The detector-averaged RVS for MS 1 of Terra MODIS bands 10, 13, and 17 at the AOI of the SV, generated using Approach I, is shown in Fig. 17. It can be clearly seen that the onorbit change in the RVS for the shorter wavelength band 10 $(0.488 \mu \mathrm{m})$ is about $17 \%$, whereas the RVS changes for bands $13(0.667 \mu \mathrm{m})$ and $17(0.905 \mu \mathrm{m})$ are about $3 \%$ and less than $1 \%$, respectively. The largest MS differences of the RVS are about $8 \%$ for band $10(0.488 \mu \mathrm{m})$ and less than $1 \%$ for the other two bands. The Aqua MODIS RVS trends for bands 10, 13, and 17 shown in Fig. 18 also show the shorter wavelength band 10 exhibiting a larger change compared to bands 13 and 17 while the MS differences for all three bands are much smaller compared to the corresponding Terra bands. For Aqua bands 13 and 17, the RVS at the AOI of the SV has generally increased with time, in contrast with Aqua band $10(0.488 \mu \mathrm{m})$ and Terra bands 10, 13, and 17. As mentioned in Section II, the RVS is normalized at the AOI of the SD, and the RVS at the AOI of the SV is the gain difference between the AOI of the SV and that of the SD. Thus, the increase of the RVS at the SV does not necessarily mean that the gain at that AOI increases.

Fig. 19 shows the Terra band $8(0.412 \mu \mathrm{m})$ detector-averaged RVS derived from the EV and lunar response trending for MS 1 at four representative AOIs using Approach II, which are denoted by solid symbols. The RVS is normalized at the $\mathrm{AOI}$ of the SD. At the AOI of the SV, the RVS decreases

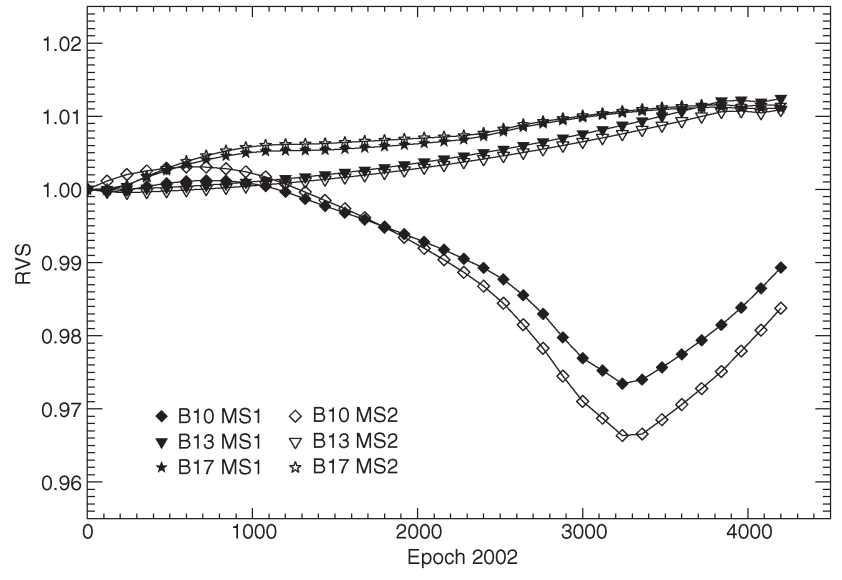

Fig. 18. RVS for Aqua MODIS bands 10, 13, and 17 at the AOI of the SV.

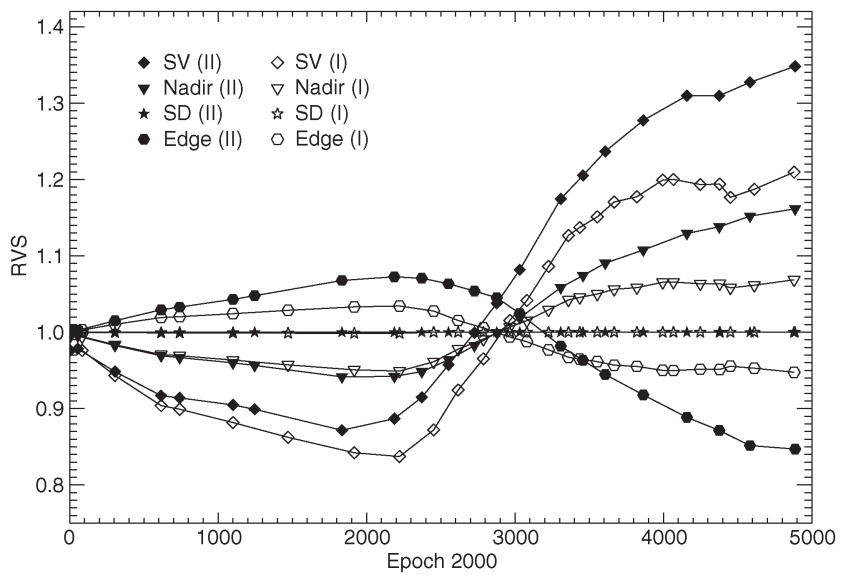

Fig. 19. RVS for Terra MODIS Band 8 MS 1. Unfilled symbols: Approach I. Solid symbols: Approach II.

about $10 \%$ in the first five years on orbit, then increases about $47 \%$ in the following eight years, and exhibits a steady change thereafter. The change in the RVS at the SV is representative of the difference in the rate of gain changes derived from the SD and lunar observations. This observation is also evident in the relative RVS change presented in Fig. 16. For comparison, the RVS generated using Approach I is also plotted and denoted by unfilled symbols in the figure. The differences between the two sets of RVS essentially represent the differences between C5 and C6 since the former is based on Approach I. In general, a temporal increase at all AOIs is observed, with the largest change of around $13 \%$ observed at the AOI of the SV. The large RVS difference at the AOI of the SV does not imply a large difference in the retrieved reflectance between the two versions.

The MODIS L1B EV reflectance or radiance is proportional to $m_{1} /$ RVS, which is inversely proportional to the gain of the band at any given AOI. Fig. 20 shows the corresponding $m_{1} /$ RVS trending for Terra band 8 MS 1 at the four representative AOIs using the RVS derived from both approaches. The difference between the two sets of $m_{1} /$ RVS are expectedly negligible at the AOI of the SV since the on-orbit gain change at the SV AOI is primarily determined by the lunar calibration. The differences vary with AOI and increase with time, particularly after the designed lifetime of six years. Currently, the differences 


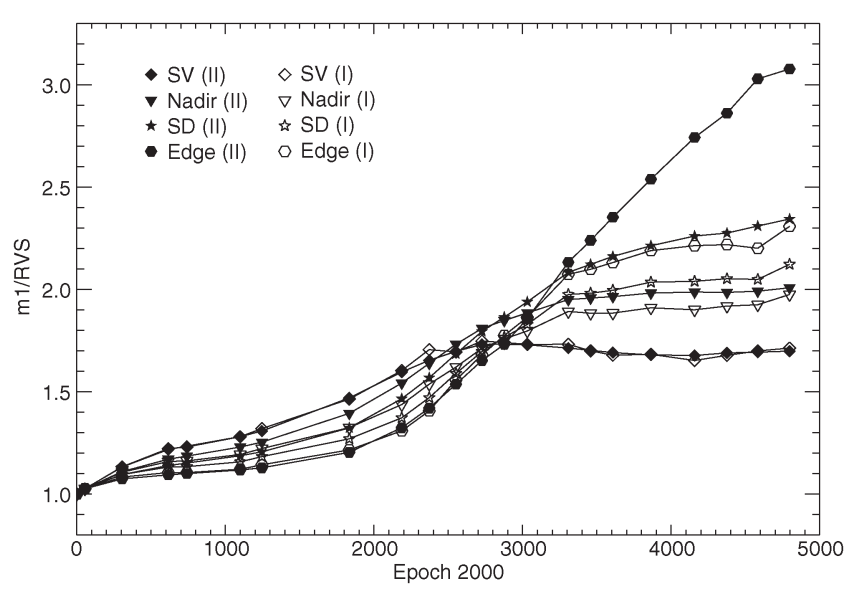

Fig. 20. Normalized $m_{1} /$ RVS for Terra MODIS band 8 MS 1. Unfilled symbols: Approach I. Solid symbols: Approach II.

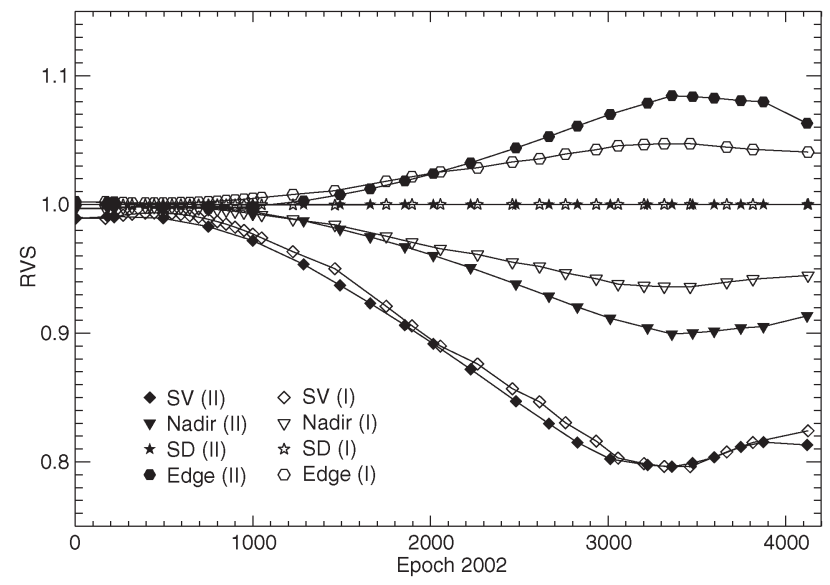

Fig. 21. Normalized RVS for Aqua MODIS Band 8 MS 1. Unfilled symbols: Approach I. Solid symbols: Approach II.

at the AOI of the nadir, SD, and end of scan of large AOI are about $5 \%, 12 \%$, and $23 \%$, respectively. The gain difference at the SD AOI indicates an increased uncertainty of the SD calibration for band $8(0.412 \mu \mathrm{m})$. The differences of the RVS at other AOIs indicate that the linear approximation for the AOI dependence of the RVS is not adequate to accurately describe the on-orbit change. Consequently, EV response trending has to be used to derive the time-dependent gain and RVS for Terra band 8. Similar behavior is also observed for other Approach II bands summarized in Table II.

Fig. 21 shows the detector-averaged RVS trends from both approaches for Aqua band 8 MS 1 at the four representative AOIs. Also, as expected, there is no difference between the two approaches at the AOI of the SD. At the AOI of the SV, the difference is less than $1 \%$, much smaller compared to that observed for Terra band $8(0.412 \mu \mathrm{m})$. Thus, the small difference between the approaches at the AOI of the SV indicates that the bidirectional-reflectance-factor [26] degradation-corrected $\mathrm{SD}$ and EV responses agree reasonably well. However, the differences at the AOI of the nadir and at the end of the scan are evident, further confirming the inadequacy of the linear approximation in characterizing the on-orbit RVS change. A similar result is also obtained for Aqua band $9(0.443 \mu \mathrm{m})$.

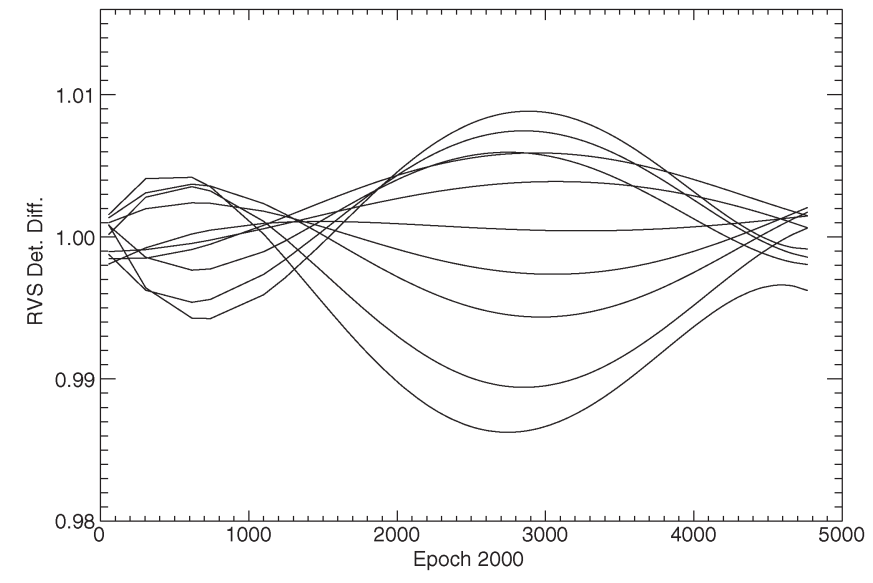

Fig. 22. Terra MODIS band 8 MS 1 RVS detector differences.

The MODIS RSB time-dependent RVS is, in principle, detector as well as band, MS, and time dependent as discussed in Section II. However, for most bands, the detector dependence is not evident, and detector-independent RVS is calculated except for short-wavelength bands that include Terra bands 3 and 8-12 and Aqua bands 8-12. Fig. 22 shows the Terra band 8 MS 1 RVS detector differences. Variations with time can be as large as $2.4 \%$. For Terra band $8 \mathrm{MS} 2 \mathrm{RVS}$, the detector differences are about the same as those for MS 1. For Terra bands 3 and 9 , the largest differences are $1.3 \%$ and $2.6 \%$, respectively. For all other Terra and Aqua bands which have detector-dependent RVS, the differences are less than $1 \%$.

Terra and Aqua band $8(0.412 \mu \mathrm{m})$ MS 1 RVS on-orbit changes since launch can be as large as 35\% and 20\%, respectively, as demonstrated in Figs. 19 and 21. For MS 2, the largest RVS changes are $28 \%$ and $19 \%$ for Terra and Aqua band 8, respectively. Apparently, Terra band 8 has a large MS difference while the Aqua band $8 \mathrm{MS}$ difference is very small. For Terra band 8 , the RVS on-orbit variation is even larger and can be as large as $47 \%$ and $38 \%$ for MSs 1 and 2, respectively, at small AOI since it decreased during the first few years on orbit and then sharply increased in the following years. For Terra bands $9(0.443 \mu \mathrm{m}), 3(0.469 \mu \mathrm{m}), 10(0.488 \mu \mathrm{m}), 11(0.531 \mu \mathrm{m})$, $12(0.551 \mu \mathrm{m})$, and $4(0.555 \mu \mathrm{m}) \mathrm{MS} 1$, the largest on-orbit RVS changes are about $16 \%, 13 \%, 18 \%, 11 \%, 7 \%$, and $4 \%$, respectively, while for MS 2, the largest RVS changes for these bands are about $25 \%, 12 \%, 15 \%, 13 \%, 7 \%$, and $6 \%$, respectively. The RVS on-orbit variation is even larger for Terra band 9 MSs 1 and 2 and band 3 mirror side 2 with variations reaching $30 \%, 35 \%$, and $28 \%$, respectively. MS differences are clearly seen for Terra bands 9, 3, 10, and 4. For Aqua MODIS, the RVS MS difference is much smaller for all RSBs (within $1 \%$ or less) compared to Terra MODIS RSB, and the on-orbit RVS change is also relatively smaller. For Aqua bands 9, 3, 10, $1(0.645 \mu \mathrm{m})$, and $2(0.858 \mu \mathrm{m})$, the largest changes are about $13 \%, 5 \%, 3 \%, 3 \%$, and 3\%, respectively. For all other Terra and Aqua RSBs, the on-orbit RVS changes are about $2 \%$ or within $2 \%$. For the SWIR bands, the on-orbit RVS change is not noticeable, and thus, the prelaunch RVS is applied as mentioned in Section II. Considering that the uncertainty specification for MODIS RSB EV reflectance is 2\%, the time-dependent RVS is 


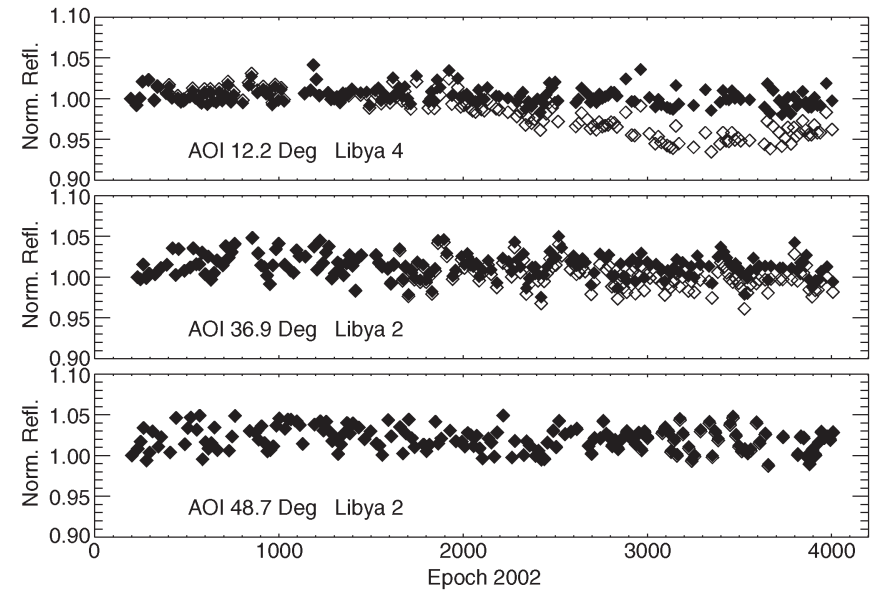

Fig. 23. Aqua band 3 EV reflectance trending. Solid: Time-dependent RVS. Unfilled: Prelaunch RVS.

critical and essential for most of the MODIS RSB, particularly for the short-wavelength bands.

Currently, six Terra bands and two Aqua bands need to use the desert response trending to track on-orbit RVS change while the other MODIS RSBs only need SD and lunar calibration to accurately derive their time-dependent RVS. As both instruments age with passing time, either the SD calibration has become inadequate to catch the gain change at the AOI of the $\mathrm{SD}$ or the linear approximation for the AOI dependence for the on-orbit RVS change has become invalid for some of the bands. Consequently, Approach II has to be applied in these bands to derive the time-dependent RVS as previously done for Terra bands 1-4, 8, and 9 and Aqua bands 8 and 9. For most ocean bands, saturation occurs when viewing desert sites, and hence, ocean sites may be used to track the response change. However, the response trending over ocean sites are much noisier, and thus, it is challenging to apply Approach II to other ocean bands. For Terra bands 3 and 8-9, quite accurate timedependent RVS has been derived from the response trending over desert sites as well as from the measurements of the onboard calibrators, but it remains a challenge to reduce the polarization effect at the end of the scan and the large AOI, particularly for forward LUT updates.

\section{B. EV Reflectance Trending Over Desert Sites}

The EV reflectance trending over the pseudo-invariant sites can be used to access the performance of the time-dependent RVS. Fig. 23 shows the BRDF-corrected TOA reflectance observed over two desert sites, Libya 2 and Libya 4, at three selected AOIs for MS 1 of Aqua MODIS band $3(0.469 \mu \mathrm{m})$. The three selected AOIs are $12.2^{\circ}, 36.9^{\circ}$, and $48.7^{\circ}$, representing the AOI of the SV, nadir, and SD, respectively. The reflectance trending in Fig. 23 has been normalized to the first measurement. The TOA reflectance trends generated using C6 on-orbit calibration coefficients $m_{1}$ and prelaunch RVS are denoted using open circles, and those generated using C6 on-orbit calibration coefficients and on-orbit RVS are denoted using solid circles. Based on the assumption that the desert targets are temporally stable, no long-term drift is expected in

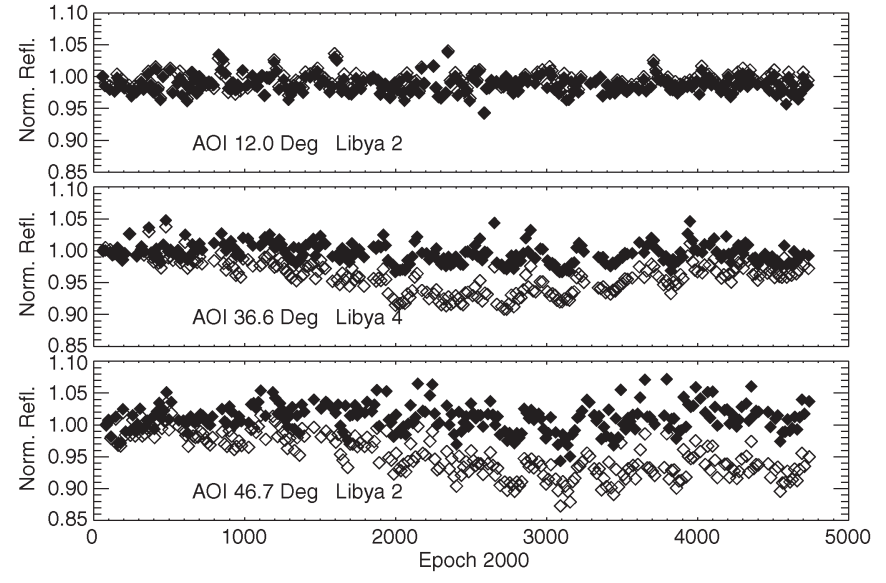

Fig. 24. TOA reflectance trending for Terra MODIS band 8 MS 1. Unfilled: Approach I $m_{1}$ and RVS applied. Solid: Approach II $m_{1}$ and RVS.

the reflectance trending, provided that the band is accurately calibrated. As expected, no evidence of drifts is seen at the AOI of $48.7^{\circ}$ in the reflectance trending from either data sets since the on-orbit SD calibration is dominant in characterizing the gain change at this AOI. For the other two frames, the reflectance trending generated using prelaunch RVS exhibits a significant long-term drift, particularly after four years on orbit. The drifts accumulate to $2 \%$ to $6 \%$ at AOIs of $36.9^{\circ}$ and $12.2^{\circ}$, respectively. The above result highlights the necessity of a time-dependent RVS to accurately characterize the onorbit sensor change at all AOIs for Aqua band $3(0.469 \mu \mathrm{m})$. It is demonstrated that SD/SDSM and lunar calibration can accurately characterize the on-orbit gain changes at the AOI of the SD and SV and that the linear approximation for the AOI dependence of the on-orbit RVS change works quite well for the band. A similar conclusion can also be reached for other Approach I bands from the reflectance trending at the pseudoinvariant sites using unsaturated data.

The pseudo-invariant desert sites can be used to demonstrate if Approach II is necessary for deriving the time-dependent RVS for a MODIS RSB while the site data are not saturated. Fig. 24 shows the BRDF-corrected TOA reflectance observed over two desert sites, Libya 2 and Libya 4, at three selected AOIs, $12.0^{\circ}, 36.6^{\circ}$, and $46.7^{\circ}$, for Terra band $8 \mathrm{MS} 1$. The chosen frames represent the SV, nadir, and SD AOI, respectively. The unfilled symbols denote the reflectance generated from Approach I, and the filled symbols denote the Approach II results. At the AOI of $12.0^{\circ}$, the observed long-term drift is generally within the calibration uncertainty specification of $2 \%$. This also indicates that the on-orbit lunar calibration provides an accurate characterization of the instrument gain. The approximately $10 \%$ long-term drift observed from the reflectance trending at the SD AOI further confirms the inadequacy of the $\mathrm{SD}$ to characterize the on-orbit gain change accurately. For the AOI of $36.6^{\circ}$, the unfilled symbols denote the reflectance calculated using Approach I which shows a decrease (up to 10\%) for the first seven years followed by an increase in the trend thereafter. In comparison to the long-term drift observed at AOIs of $36.6^{\circ}$ and $46.7^{\circ}$, the nonlinear dependence of the RVS as a function of AOI can be clearly seen. The solid symbols denote the reflectance trending computed using Approach II 


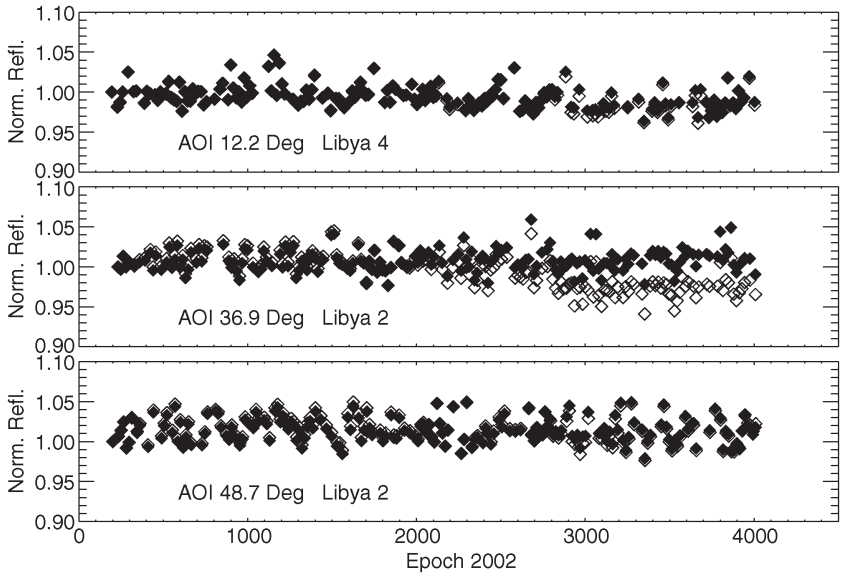

Fig. 25. TOA reflectance trending for Terra MODIS band 8 MS 1. Unfilled: Approach I $m_{1}$ and RVS applied. Solid: Approach II $m_{1}$ and RVS.

which shows a clear mitigation of the long-term drifts observed in Approach I. Thus, Approach II essentially is required in order to derive an accurate time-dependent RVS. A similar conclusion can be reached for Terra bands $1-4$ and 9 .

Fig. 25 shows the BRDF-corrected TOA reflectance observed at the desert sites, Libya 2 and Libya 4, for MS 1 of Aqua band 8. Similar to Fig. 24, the unfilled symbols denote the reflectance generated using Approach I, and the filled symbols denote the reflectance produced using Approach II. At AOIs of $12.2^{\circ}$ and $48.7^{\circ}$, the observed long-term drift is generally seen to be within the calibration uncertainty specification, which further reaffirms the ability of the onboard SD and lunar measurements to accurately catch the on-orbit gain change at the $\mathrm{AOI}$ of the SD and that of the SV. A long-term drift of about $5 \%$ is observed in the reflectance trending denoted by open circles at an AOI of $36.9^{\circ}$. This confirms that the linear approximation for the AOI dependence of the RVS onorbit change is inadequate for Aqua band 8 and justifies the usage of response trending from EV targets to supplement the onboard measurements. The corresponding Approach IIderived reflectance trends show that the long-term drifts are significantly mitigated, and thus, Approach II is necessary for deriving an accurate time-dependent RVS for Aqua band 8. A similar result is also observed for Aqua band 9. An independent approach for correcting the long-term drift in Aqua MODIS RSB was developed by Meister et al. and a similar improvement was reported in bands 8 and 9 [28].

The EV reflectance can also be used to assess the necessity of applying the RVS detector dependence. Fig. 26 shows the differences of the EV reflectance observed by different detectors of MS 1 of Terra band $8(0.412 \mu \mathrm{m})$ over the ocean site in MODIS C5 and C6, where the detector-independent and the detector-dependent RVS are applied, respectively. The TOA reflectance difference of each detector relative to the mean at the AOI of the nadir is shown in the figure. The top plot in the figure shows that there are clear differences among the reflectance observed by different detectors in Terra band 8 C5 $\mathrm{EV}$ products. The differences vary with time and can be as large as $\pm 2 \%$. The bottom plot in the figure shows that the detector differences in Terra band 8 C6 EV reflectance products are re-

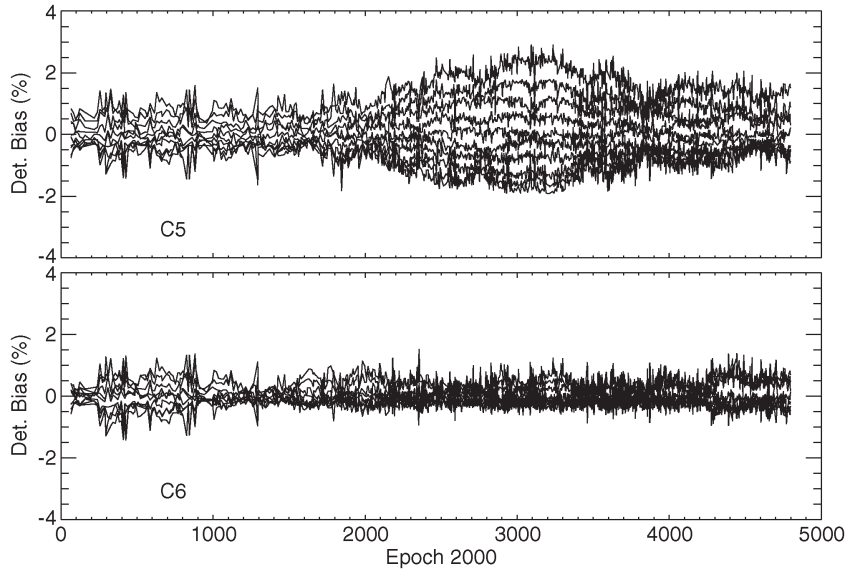

Fig. 26. Terra band 8 C5 and C6 detector differences at the AOI of the SV.

duced to within $\pm 1 \%$. A similar conclusion can also be reached for other bands whose RVSs are also detector dependent.

The total uncertainty of the RVS derived using either approaches depends on the uncertainties of input measurements and the associated assumptions. In addition to the detector dependence at short wavelengths, the uncertainty is primarily band, MS, and AOI dependent. Preliminary analysis shows that the uncertainty of the RVS derived using the first approach (onboard calibrators) at the AOI of the SV is generally within $2 \%$ [24]. The uncertainty of the RVS at the AOI of the SD is estimated to be smaller than that at the AOI of the SV since the RVS is normalized at the AOI of the SD. The uncertainty of the RVS derived using the second approach is estimated to be greater than $2 \%$, particularly at large AOI due to the impact of the polarization [24]. In the case of Terra band 8 , the uncertainty of the RVS varies between $2 \%$ and $4 \%$. A comprehensive uncertainty analysis of the MODIS RSB calibration will be discussed in detail elsewhere.

\section{CONCLusion}

Some RSBs of both Terra and Aqua MODIS instruments have experienced significant degradation after more than 10 years on orbit. The magnitude of the on-orbit degradation is more predominant in the case of Terra MODIS RSB, primarily due to the smoking incident prior to launch and the increased SD degradation since July 2, 2003. Prelaunch characterization exhibited a wavelength dependence in the RVS; with the largest change observed at the shorter wavelengths. The observed degradation differences at different AOIs over time are corrected using time-dependent RVS LUTs in the L1B calibration. Two approaches have been developed and applied to calculate the $\mathrm{C} 6$ time-dependent on-orbit RVS change. The first approach tracks the on-orbit RVS change using the SD and lunar observations and EV MS ratios of the instrument response. This approach has been applied to derive the time-dependent RVS for Terra bands 10-19 and Aqua bands 1-4 and 10-19. For most of these bands, the RVS on-orbit change is within 5\% with the exception of Terra bands 10-12 where it is seen to be as large as $20 \%$. Monitoring of the pseudoinvariant desert targets indicates a reasonable multiyear performance with no observable longterm drift. A second approach is developed to update the 
on-orbit RVS change by supplementing the lunar measurements with the EV response trending from pseudoinvariant desert targets. This approach is used to track the on-orbit RVS change for the selected short-wavelength RSBs, Terra bands 1-4, 8, and 9 and Aqua bands 8 and 9. The RVS for these bands have exhibited a significant on-orbit change, about $35 \%$ for Terra band 8 MS 1 and $20 \%$ for Aqua band 8 MS 1. In the case of Terra band 8 , the difference between the two approaches of the RVS derivation exhibits a difference of $13 \%$ at the AOI of the SV. In addition to the wavelength dependence, the on-orbit RVS change for Terra MODIS has also indicated a MS and a detector dependence primarily in the short-wavelength bands. For Aqua MODIS RVS, both the MS and detector differences are seen to be comparatively within $2 \%$ for band 8 and generally less than $1 \%$ for other RSBs. An accurate characterization of the time-dependent RVS is vital in maintaining the accuracy of the MODIS L1B products. The lessons learned from the MODIS on-orbit RVS behavior can serve as a useful reference for future Earth-observing sensors.

\section{ACKNOWLEDGMENT}

The authors would like to thank G. Toller, M. Chu, A. Wald, and $\mathrm{B}$. Wenny for their valuable comments and suggestions.

\section{REFERENCES}

[1] W. L. Barnes and V. V. Salomonson, "MODIS: A global image spectroradiometer for the Earth Observing System," Crit. Rev. Opt. Sci. Technol., vol. CR47, pp. 285-307, 1993.

[2] W. L. Barnes, V. V. Salomonson, B. Guenther, and X. Xiong, "Development, characterization, and performance of the EOS MODIS sensors," in Proc. SPIE, 2003, vol. 5151, pp. 337-345.

[3] W. L. Barnes, X. Xiong, and V. V. Salomonson, "Status of Terra MODIS and Aqua MODIS," J. Adv. Space Res., vol. 32, no. 11, pp. 2099-2106, Dec. 2003.

[4] V. V. Salomonson, W. L. Barnes, X. Xiong, S. Kempler, and E. Masuoka, "An overview of the Earth Observing System MODIS instrument and associated data systems performance," in Proc. IEEE IGARSS, 2002, pp. 1174-1176.

[5] X. Xiong and W. L. Barnes, "An overview of MODIS radiometric calibration and characterization," Adv. Atmos. Sci., vol. 23, no. 1, pp. 69-79, Jan. 2006.

[6] X. Xiong, B. N. Wenny, and W. L. Barnes, "Overview of NASA Earth Observing Systems Terra and Aqua moderate resolution imaging spectroradiometer instrument calibration algorithms and on-orbit performance," J. Appl. Remote Sens., vol. 3, no. 1, p. 032501, Jun. 2009.

[7] X. Xiong, J. Sun, J. Esposito, B. Guether, and W. L. Barnes, "MODIS reflective solar bands calibration algorithm and on-orbit performance," in Proc. SPIE, 2002, vol. 4891, pp. 95-104.

[8] X. Xiong, J. Sun, W. Barnes, V. Salomonson, J. Esposito, H. Erives, and B. Guenther, "Multi-year on-orbit calibration and performance of Terra MODIS reflective solar bands," IEEE Trans. Geosci. Remote Sens., vol. 45, no. 4, pp. 879-889, Apr. 2007.

[9] X. Xiong, J. Sun, X. Xie, W. Barnes, and V. Salomonson, "On-orbit calibration and performance of Aqua MODIS reflective solar bands," IEEE Trans. Geosci. Remote Sens., vol. 48, no. 1, pp. 535-546, Jan. 2010.

[10] X. Xiong, K. Chiang, A. Wu, W. Barnes, B. Guenther, and V. Salomonson, "Multiyear on-orbit calibration and performance of Terra MODIS thermal emissive bands," IEEE Trans. Geosci. Remote Sens., vol. 46, no. 6, pp. 1790-1803, Jun. 2008.

[11] X. Xiong, B. Wenny, A. Wu, W. L. Barnes, and V. Salomonson, "Aqua MODIS thermal emissive bands on-orbit calibration, characterization, and performance," IEEE Trans. Geosci. Remote Sens., vol. 47, no. 3, pp. 803814, Mar. 2009.

[12] X. Xiong, N. Che, and W. L. Barnes, "Terra MODIS on-orbit spatial characterization and performance," IEEE Trans. Geosci. Remote Sens., vol. 43, no. 2, pp. 355-365, Feb. 2005.
[13] X. Xiong, N. Che, and W. L. Barnes, "Terra MODIS on-orbit spectral characterization and performance," IEEE Trans. Geosci. Remote Sens., vol. 44, no. 8, pp. 2198-2206, Aug. 2006.

[14] X. Xiong, R. Wolfe, W. Barnes, B. Guenther, E. Vermote, N. Saleous, and V. Salomonson, "Terra and Aqua MODIS design, radiometry, and geometry in support of land remote sensing," Land Remote Sens. Glob. Environ. Change, Remote Sens. Digital Image Process., vol. 11, pt. 2, pp. 133-164, 2011.

[15] W. L. Barnes, T. S. Pagano, and V. V. Salomonson, "Prelaunch characteristics of the Moderate Resolution Imaging Spectroradiometer (MODIS) on EOS-AM1," IEEE Trans. Geosci. Remote Sens., vol. 36, no. 4, pp. 10881100, Jul. 1998.

[16] X. Xiong, N. Che, C. Pan, X. Xie, J. Sun, W. Barnes, and B. Guenther, "Results and lessons from MODIS reflective solar bands calibration: Prelaunch to on-orbit," in Proc. SPIE, 2006, vol. 6296, p. 629607.

[17] C. Pan, X. Xiong, and N. Che, "MODIS pre-launch characterization of reflective solar band response vs. scan angle," in Proc. SPIE, 2007, vol. 6677, p. 66770R

[18] J. Sun, X. Xiong, W. L. Barnes, B. Guenther, and X. Xiong, "MODIS reflective solar bands on-orbit lunar calibration," IEEE Trans. Geosci. Remote Sens., vol. 45, no. 7, pp. 2383-2393, Jul. 2007.

[19] X. Xiong, J. Sun, and W. Barnes, "Intercomparison of on-orbit calibration consistency between Terra and Aqua MODIS reflective solar bands using the moon," IEEE Geosci. Remote Sens. Lett., vol. 5, no. 4, pp. 778-782, Oct. 2008.

[20] X. Geng, A. Angal, J. Sun, A. Wu, T. Choi, and X. Xiong, "Characterization of MODIS mirror side difference in the reflective solar spectral region," in Proc. SPIE, 2011, vol. 8153, pp. 815 31O-1-81531O-9.

[21] H. Cosnefroy, M. Leroy, and X. Briottet, "Selection and characterization of Saharan and Arabian desert sites for the calibration of optical satellite sensors," Remote Sens. Environ., vol. 58, no. 1, pp. 101-114, Oct. 1996.

[22] J. Sun, X. Xiong, A. Angal, H. Chen, X. Geng, and A. Wu, "On-orbit performance of the MODIS reflective solar bands time-dependent response versus scan angle algorithm," in Proc. SPIE, 2012, vol. 8510, p. 85100J.

[23] J. Sun, X. Xiong, H. Chen, A. Angal, A. Wu, and X. Geng, "Timedependent response versus scan angle for MODIS reflective solar bands," in Proc. SPIE, 2009, vol. 7452, pp. 745219-1-745219-11.

[24] J. Sun, X. Xiong, H. Chen, A. Angal, X. Geng, and A. Wu, "Time-dependent response versus scan angle and its uncertainty for MODIS reflective solar bands," in Proc. SPIE, 2010, vol. 7826, pp. 782620-1-782620-12.

[25] J. L. Roujean, M. J. Leroy, and P. Y. Deschamps, "A bidirectional reflectance model of the Earth's surface for the correction of remote sensing data," J. Geophys. Res., vol. 97, no. D18, pp. 20 455-20 468, Dec. 1992.

[26] J. Sun and X. Xiong, "MODIS polarization sensitivity analysis," IEEE Trans. Geosci. Remote Sens., vol. 45, no. 9, pp. 2875-2885, Sep. 2007.

[27] E. Kwiatkowska, B. A. Franz, G. Meister, C. McClain, and X. Xiong, "Cross calibration of ocean-color bands from moderate resolution imaging spectroradiometer on Terra platform," Appl. Opt., vol. 47, no. 36, pp. 6796-6810, Dec. 2008.

[28] G. Meister, B. A. Franz, E. J. Kwiatkowska, and C. R. McClain, "Corrections to the calibration of MODIS Aqua ocean color bands derived from SeaWiFS data," IEEE Trans. Geosci. Remote Sens., vol. 50, no. 1, pp. 310-319, Jan. 2012.

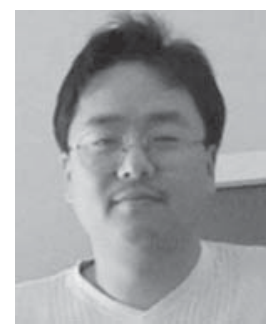

Junqiang Sun received the B.S. degree in physics from the Changsha Institute of Technology, Changsha, China, the Ph.D. degree in physics from the University of Science and Technology of China, Hefei, China, and the Ph.D. degree in chemistry from the University of Florida, Gainesville, FL, USA.

$\mathrm{He}$ is currently a Chief Scientist with Sigma Space Corporation, Lanham, MD, USA, serving as the Technical Leader for the Moderate Resolution Imaging Spectroradiometer (MODIS) Characterization Support Team. He is also involved with the onorbit calibration and characterization of the Suomi National Polar-orbiting Partnership (SNPP) - Visible Infrared Imaging Radiometer Suite (VIIRS). Before joining Sigma Space Corporation, he was a Lead Scientist with Science Systems and Applications, Inc., Lanham, MD, USA, and worked on MODIS and VIIRS calibration and characterization. He has also extensively worked in atomic and molecular physics at Freie Universitaet Berlin, Berlin, Germany, and Kansas State University, Manhattan, KS, USA, and in quantum chemistry at Iowa State University, Ames, IA, USA, and the University of Florida. 


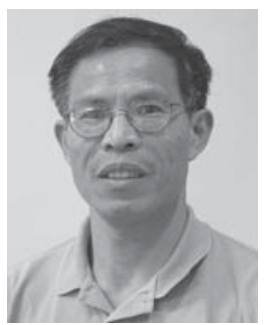

Xiaoxiong Xiong received the B.S. degree in optical engineering from the Beijing Institute of Technology, Beijing, China, and the Ph.D. degree in physics from the University of Maryland, College Park, MD, USA.

Before joining the NASA/Goddard Space Flight Center (GSFC), he had also worked in the fields of optical instrumentation, nonlinear optics, laser/ atomic spectroscopy, and mass spectrometry at private industry and at the National Institute of Standards and Technology. He is an Optical Physicist at NASA GSFC, working on the Earth Observing System (EOS) and the Suomi National Polar-orbiting Partnership (SNPP) Visible Infrared Imaging Radiometer Suite (VIIRS) sensor calibration and characterization. He is currently serving as the Moderate Resolution Imaging Spectroradiometer (MODIS) Project Scientist for the instrument operation and calibration and the Technical Lead for both the MODIS Characterization Support Team and the NPP Instrument Calibration Support Team.

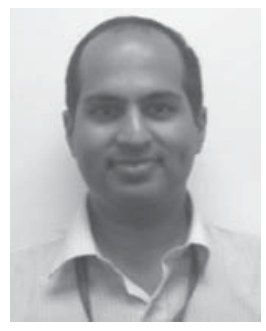

Amit Angal received the M.S degree in electrical engineering from South Dakota State University, Brookings, SD, USA.

He is currently a Senior Instrument Engineer with Science Systems and Applications, Inc., Lanham, $\mathrm{MD}$, USA, primarily working on the radiometric characterization and calibration of the reflective solar bands of the Moderate Resolution Imaging Spectroradiometer (MODIS) instruments onboard the Terra and Aqua spacecraft. His research interests include sensor cross-calibration and validation.

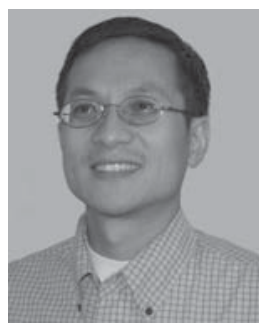

calibrations.

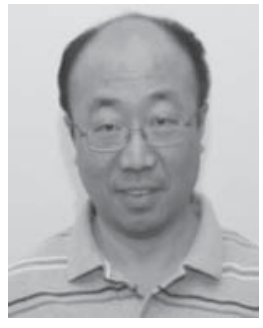

Space Corporation, Lanham, MD, USA

Xu Geng received the B.S. degree in engineering mechanics from Tsinghua University, Beijing, China, and the Ph.D. degree in Mechanical Engineering from Johns Hopkins University, Baltimore, MD, USA.

He is currently working on the instrument calibration and data analysis with the Moderate Resolution Imaging Spectroradiometer Characterization Support Team. He is currently with Sigma Space Corporation, Lanham, MD, USA.

Hongda Chen received the B.S. and M.S. degrees in electrical engineering from the Beijing Institute of Technology, Beijing, China, in 1987 and 1990, espectively, and the Ph.D. degree in information echnology from George Mason University, Fairfax, 1999.

He currently serves as a Senior Research Scientist rigna Space Corporation, Lanham, MD, USA, ests include signal processing, data analysis, communications, fault diagnosis/prognosis, and instrument

Aisheng $\mathbf{W u}$ received the B.S. degree in physics and atmospheric science from the University of Science and Technology of China, Hefei, China, the M.S. degree in atmospheric remote sensing from the Institute Plateau Atmospheric Physics, Chinese Academy Science, Lanzhou, China, and the Ph.D. degree in Columbia, Vancouver, Canada.

He is currently a Senior Scientist with the Moderate Resolution Imaging Spectroradiometer and VIIRS Characterization and Support Teams, Sigma 